\title{
La dialéctica de trabajo, valor y precio en la conceptualización del capital de Marx: una reconstrucción*
}

\author{
Mario L. Robles Báez**y Roberto Escorcia Romo***
}

\section{RESUMEN}

Dado que el objeto de la obra cimera de Marx es el concepto de capital como una totalidad, cuyo método de presentación está basado en la dialéctica sistemática, el objetivo de este trabajo es una reconstrucción de la determinación del valor social de las mercancías en cuanto productos de capital a lo largo de los pasos que conforman la estructura lógica de El capital. Se argumenta que los tiempos de trabajo social abstracto que los valores sociales de las mercancías representan definitivamente (el así llamado problema de la reducción) sólo pueden ser plenamente puestos y actualizados en el momento en que los precios de producción de las mercancías son determinados (el así llamado problema de la transformación). Tras esta reconstrucción, es posible fundamentar regresivamente el punto de partida de la presentación y concebir la mercancía como una unidad de múltiples determinaciones.

Palabras clave: trabajo, forma-valor, dinero, precio, concepto marxista del capital, dialéctica sistemática: Hegel y Marx.

Clasificación JEL: B51, P16.

\section{AbstraCT}

Considering that the object of most important Marx's work is the concept of capital as a totality, whose method of presentation is based on systematic dialectics, the aim of this paper is a reconstruction of the determination of the social value of commodities as products of capital throughout the moments conforming the logical structure of Capital. It is argued that the social abstract-labour time that the values of commodities definitively represent (the so-called reduction problem) can only be fully posited and actualized up to the moment in which commodities's prices of production are determined (the so-called transformation problem). It is only at the the end of this presentation that the point of departure of the presentation can be retrogressive grounded and the commodity can be conceptualized as a unity of multiple determinations.

Keywords: labour, value-form, money, price, marxist concept of capital, systematic dialectics: Hegel and Marx.

JEL classification: B51, P16.

\footnotetext{
* Fecha de recepción: 23/11/2012. Fecha de aprobación: 09/09/2014.

** Profesor titular del Departamento de Producción Económica de la Universidad Autónoma Metropolitana-Xochimilco. Tel.: 5483-7100. Correo electrónico: robm6308@correo.xoc.uam.mx.

*** Profesor asociado del Departamento de Producción Económica de la Universidad Autónoma Metropolitana-Xochimilco. Correo electrónico: escortarex@gmail.com.
} 


\section{INTRODUCCIÓN}

Para Marx, el capital es el sujeto económico de la sociedad capitalista. ${ }^{1}$ La conceptualización de éste como una totalidad, basada metodológicamente en un desarrollo de la dialéctica sistemática de Hegel, ${ }^{2}$ es presentada en El capital. Esto es, el concepto de capital es presentado como una totalidad que resulta de su propio movimiento dialéctico de determinación, ${ }^{3}$ un movimiento en el que todas sus formas necesarias de existencia (o momentos) que constituyen su estructura lógica interna de presentación están sistemáticamente ordenados e interrelacionados en forma sincrónica, adquiriendo progresivamente un mayor grado de concreción y determinación. A partir del momento inmediato que corresponde a las categorías representativas de las formas de existencia más simples y abstractas del capital, el argumento dialéctico de Marx se mueve progresivamente hacia

\footnotetext{
${ }^{1}$ En El capital, lo define como "valor que se valoriza a sí mismo" y, en cuanto tal, lo caracteriza como "un sujeto automático", "el sujeto de un proceso", "sujeto dominante", "una sustancia en proceso, dotada de movimiento propio", "valor en proceso, dinero en proceso" (Marx, 1982a, pp. 188-189). A su vez, en los Grundrisse, lo caracteriza como "la potencia económica, que lo domina todo, de la sociedad burguesa. Debe constituir el punto de partida y el punto de llegada [....]" (Marx, 1984a, p. 28)

2 "En el método de elaboración de la cuestión, algo me ha prestado un gran servicio: by mere accident había ojeado de nuevo la Lógica de Hegel...” (Marx y Engels, 1983, p. 86). Sobre su posición ante el método dialéctico de Hegel, Marx señala: "Mi método dialéctico no sólo difiere del de Hegel, en cuanto a sus fundamentos, sino en que es su antítesis directa. Para Hegel, el proceso del pensar, al que convierte incluso, bajo el nombre de idea, en un sujeto autónomo, es el demiurgo de lo real; lo real no es más que su manifestación externa. Para mí, a la inversa, lo ideal no es sino lo material transpuesto y traducido en la mente humana... La mistificación que sufre la dialéctica en manos de Hegel, en modo alguno obsta para que haya sido él quien, por primera vez, expuso de manera amplia y consciente las normas generales del movimiento de aquélla. En él, la dialéctica está puesta al revés. Es necesario darle vuelta, para descubrir así el núcleo racional que se oculta bajo la envoltura mística." (Marx, 1982a, pp. 19-20).

${ }^{3}$ Por el hecho de conceptualizar el capital como sujeto y que su método de presentación sea la dialéctica sistemática, Marx ha sido calificado de metafísico por marxistas y no marxistas. Por ejemplo, J. Shumpeter pretende reducir el método dialéctico de Marx al de la ciencia positiva: "Marx no traicionó jamás la ciencia positiva con la metafísica" (citado por Pietranera, 1977, p. 28). Por su parte, J. Robinson señala que, para ella, "es imposible hablar en inglés a un marxista porque sólo comprende el 'hegelés', una lengua que nunca he dominado y que, en todo caso considero un medio de comunicación muy deficiente cuando se trata de conceptos de lógica pura" (Robinson, 1976, p. 332). En realidad, como lo señalamos en otro texto, "Marx no es metafísico, sino que es la metafísica de la realidad capitalista, es decir, una realidad invertida en la que el capital en cuanto una abstracción real como sujeto, domina y somete a los seres humanos, la que impone el método lógico apropiado para su estudio..." (Robles, 2005, pp. 23), es decir, la dialéctica sistemática, que contradice al sentido común y a la lógica formal.
} 
momentos en los cuales las categorías van adquiriendo un mayor grado de concreción y complejidad, formando una cadena secuencial de relaciones negativas internas a través de la cuales las categorías precedentes constituyen no sólo la presuposición de las que son progresivamente puestas, ${ }^{4}$ sino que también son dialécticamente negadas; en otras palabras, como las categorías son determinadas progresivamente, las precedentes no sólo se conservan, sino además se fundamentan regresivamente, probando ser así necesarias para las consecuentes. ${ }^{5}$

${ }^{4}$ Lo que es "puesto" es hecho explícito, reconocido como real o como existente, que llega a
tener una existencia socialmente determinada, en otras palabras, lo puesto es algo que ha sido so-
cialmente fundado. Así, "poner" se refiere a la afirmación de ese algo. En cuanto este algo mismo
tiene determinaciones (propiedades, cualidades, etc.) inmediatas presupuestas o implícitas por ade-
lantado (in advance) -sus presuposiciones--, su posición debe significar a su vez la posición de sus determinaciones. Es decir, las presuposiciones son en efecto puestas por el resultado aunque ellas sean puestas por adelantado. A lo largo del texto la idea de posición pone énfasis en el fundamento social (lo socialmente determinado) como principio objetivo que determina si algo existe o no. Para que el capital sea puesto, éste debe producir como resultado propio aquello (una cualidad específica) que inicialmente le era presupuesto (asumido, le era otorgado de antemano, por adelantado): valorizarse a sí mismo. En el momento de la posición, el capital afirma la existencia de sí mismo, hace explícito aquello que era implícito en él.

${ }^{5}$ El significado de negación dialéctica no es el de la negación formal según el cual una proposición es verdadera o falsa y donde una proposición y su negación no pueden ser ambas verdaderas o ambas falsas (ello implicaría una contradicción formal); la validez de una implica la falsedad de la otra. Por ejemplo, si el enunciado A es verdadero, entonces no-A (en símbolo: $\neg$ A) es falso, y viceversa. En este caso, aceptar la validez de un enunciado implica "suprimir" al otro, anularlo, abolirlo, cancelarlo, etc. Por lo contrario, la negación dialéctica se refiere a un movimiento por medio del cual un juicio se pone a sí mismo o se media consigo mismo y no a una supresión o anulación, sino, por el contrario, a una autodefinición activa a través del movimiento afirmación-negación-negación de la negación, donde la negación del juicio negado implica regresar a una afirmación, pero una afirmación diferente de aquella originalmente negada. El verbo alemán aufheben puede ser traducido como: 1) elevar, levantar, recoger; 2) anular, abolir, suprimir, cancelar, revocar, destruir, suspender; 3) mantener, guardar, conservar, preservar. En la versión inglesa, aufeheben es traducido como "subsumir" (sublate). El sustantivo aufhebung, a su vez, se traduce como "levantamiento", "abolición" y "preservación". La negación dialéctica se refiere a estos dos últimos dos significados. Hegel es explícito al respecto: "La palabra aufheben [...] tiene en el idioma [alemán] un doble sentido: significa tanto la idea de conservar, mantener, como, al mismo tiempo, la de hacer cesar, poner fin. El mismo conservar ya incluye en sí el aspecto negativo, en cuanto se saca algo de su inmediación y por lo tanto de una existencia abierta a las acciones exteriores, a fin de mantenerlo. - De este modo lo que se ha eliminado es a la vez algo conservado, que ha perdido sólo su inmediación, pero que no por esto se halla anulado - [...] Algo es eliminado sólo en cuanto ha llegado a ponerse en unidad con su opuesto; en esta determinación, más exacta que algo reflejado, puede con razón ser llamado un momento" (Hegel, 1968, pp. 97-98). En su texto sobre la lógica de Hegel, Gaete (1995, p. 16) traduce aufhebung como "negar-asumir", "negación-asunción", o por el neologismo "sursumir". Aquí se traducirá como "negar". 
Este método de presentación no corresponde, así, a una deducción analítica, que extrae de las categorías anteriores las sucesivas, sino que, como un proceso sintético, son las insuficiencias en su determinación inherentes a las categorías menos complejas las que hacen engendrar necesariamente las categorías consecuentes. ${ }^{6}$ De esta manera, el significado de las categorías cambia y se actualiza ${ }^{7}$ con la progresión de los momentos de presentación y la presentación misma continúa hasta el momento en que el concepto de capital está totalmente determinado y fundamentado como una totalidad-en-el-pensamiento, deviniendo así un sistema autosuficiente.

Sin embargo, como cualquier texto complejo, el argumento en El capital no está libre de problemas. Uno de esos problemas es la determinación del valor social de las mercancías, no como simples mercancías, sino como productos de capital, con base en el trabajo abstracto, sobre la cual el razonamiento de Marx a lo largo de los momentos en que progresivamente presenta el concepto de capital muestra ciertas ambivalencias, las cuales han dado lugar a diversas interpretaciones. Al tomar en consideración el método dialéctico sistemático de Marx delineado anteriormente y que la asignación de las categorías hegelianas utilizadas en $\mathrm{El}$ capital no se ajusta exactamente a la de la Lógica de Hegel, en este trabajo se intenta reconstruir dicha determinación, argumentando que los tiempos de trabajo social abstracto representados definitivamente por las formas de valor social de todo tipo de mercancías como productos de capital (el así llamado problema de la reducción) sólo pueden ser plenamente puestos y actualizados hasta el momento de abstracción en que sus precios de producción son determinados (el así llamado problema de la transformación) en el tomo III de El capital.

Se propone, por tanto, que dos de los problemas clásicos presentes en la literatura marxista pueden ser resueltos simultáneamente: el problema de la transformación de valores en precios de producción y el problema de la reduc-

\footnotetext{
6 "No es un método inductivo que generaliza a partir de los sucesos percibidos una ley hipotética de los fenómenos, a ser posteriormente probada en la experiencia. No es un sistema hipotético deductivo en el cual un axioma es la base de una secuencia de inferencias que formalmente lo siguen, cuyo resultado está, como se dice, ya contenido en las premisas. No es un argumento trascendental para las condiciones de posibilidad de una forma de experiencia que es considerada como ya comprobada" (Arthur, 1993, p. 67).

7 "Actualizar" y "actualizado" se refieren a la unidad de interior con lo exterior, de la esencia con la existencia, es decir, a la existencia externa de lo esencial, una externalización como reflexión. Lo actualizado es manifestación exterior y, por tanto, a través de ello, una cosa sigue un movimiento de autodiferenciación. El actualizar se presenta como contrario a la posibilidad; y, por tanto, se refiere a la capacidad de algo de "estar presente" y "ser activo".
} 
ción de los trabajos privados heterogéneos a trabajo social abstracto homogéneo. El primero de ellos se plantea alrededor de la transición de los valores de las mercancías según el tiempo de trabajo socialmente necesario (tema del tomo I de El capital) a sus precios de producción que, a partir de la competencia, deben reportar ganancias iguales a capitales iguales (asunto presente en el tomo III). Brevemente, la dificultad ha sido señalada a partir de dos hechos: el primero, que capitales de igual magnitud ponen en movimiento cantidades desiguales de trabajo, por lo que no sería posible que, según la ley del valor, obtuvieran una ganancia de igual magnitud ${ }^{8} \mathrm{y}$, segundo, que en el tercer tomo de El capital, Marx señala que las mercancías capitalistas se cambian no según su valor, sino sus precios de producción, los cuales, como resultado de la competencia, pueden ser diferentes al primero, en función de la composición orgánica de los capitales. ${ }^{9} \mathrm{La}$ pregunta consecuente se planteó sobre la "relevancia o necesidad del valor"; las respuestas posibles han sido innumerables. Dentro de ellas, nos interesa resaltar una según la cual no hay la menor relación entre valores y precios de producción, y que cuando Marx arribó al tercer tomo abandonó parcial o totalmente la ley del valor sustituyéndola por una teoría de los precios ajena a dicha ley, lo que mostraría, por tanto, la contradicción (en términos de lógica formal) interna de la

\footnotetext{
${ }^{8}$ Este punto es subrayado por Engels en el prólogo al segundo tomo de El capital: “Conforme a la ley ricardiana del valor, dos capitales que emplean la misma cantidad de trabajo vivo y lo pagan de igual manera, producen en periodos iguales -si las demás circunstancias no varían- productos del mismo valor y, asimismo, plusvalor o ganancia de igual magnitud. Pero si emplean cantidades desiguales de trabajo vivo no pueden producir plusvalor o -como dicen los ricardianos- ganancia de magnitud igual. Ahora bien, en la realidad sucede lo contrario. Capitales iguales, en efecto, independientemente de que empleen mucho o poco trabajo vivo producen, en término medio, ganancias iguales en períodos iguales. Se presenta aquí, por ende, una contradicción con la ley del valor, una contradicción ya advertida por Ricardo y que su escuela también fue incapaz de resolver. Tampoco Rodbertus pudo menos de percibir esa contradicción; en vez de resolverla, la convirtió en uno los puntos de partida de su utopía [...] Marx ya había resuelto esa contradicción en el manuscrito Contribución a la crítica ...; la solución, conforme al plan de El capital, aparece en el libro III" (Marx, 1978, pp. 22-23).

9 "La competencia (traslado o retiro de capital de una industria a otra) logra que capitales de la misma magnitud en distintas ramas, a pesar de su composición orgánica distinta [...] obtengan la misma tasa promedio de ganancia. En otras palabras: la ganancia media que un capital de $£ 100$ reporta, por ejemplo, en cierta rama, no lo hace a título de capital particular que es, ni tampoco en proporción del plusvalor que el mismo produce, sino como parte alícuota del conjunto de capital de la clase capitalista. Es una participación cuyo dividendo, proporcional a su magnitud, es sacado de la suma total del plusvalor (o trabajo no pagado) que produce el conjunto del capital variable (invertido en salarios) de la clase. [...] La competencia lleva, pues, a las mercancías no a su valor, sino a los precios de [producción], que son superiores, inferiores, o iguales a sus valores, según la composición orgánica de los capitales” (Marx y Engels, 1983, pp. 126-127) (cita corregida).
} 
construcción teórica de Marx entre el tomo i y el III. Ésta es una perspectiva compartida por autores vinculados a la tradición neoclásica y austriaca, ${ }^{10}$ a la neorricardiana $^{11}$ e incluso a la economía política marxista clásica. ${ }^{12}$ Ésta y otras interpretaciones ${ }^{13}$ son analizadas con mayor amplitud en la introducción de Robles (2011). El objetivo de este artículo consiste en mostrar que, en contra de la postura mencionada, existe una relación dialéctica entre valor y precios de producción. Un punto crucial para esto estriba en considerar que el valor no es una magnitud dada o acabada en el tomo I, con independencia de la determinación de los precios de producción en el intercambio. Esto nos coloca en el terreno del

\footnotetext{
${ }^{10}$ Por el lado de la escuela tradicional neoclásica, Paul Samuelson (1971, p. 440) indicó la existencia de dos sistemas incompatibles entre sí, el sistema de valores y el sistema de precios: "Considere dos sistemas alternativos. Escriba uno. Ahora transfórmelo tomando una goma y bórrelo. Después reemplácelo con el otro. Voilà! Ha completado su algoritmo de transformación". La imposibilidad de distinguir entre valor y precio genera que la teoría neoclásica, al rechazar la teoría del valor-trabajo, colapse el análisis de los precios de producción de Marx a uno de tipo de precios de oferta marshalianos o a una teoría ordinaria de costos de producción. Desde la posición austriaca, sostiene Böhm-Bawerk: "El tercer tomo de Marx contradice al primero. La teoría de la tasa media de ganancia y de los precios de producción no puede conciliarse con la teoría del valor. Esta es la impresión que debe, creo yo, ser recibida por todo pensador lógico" (Böhm-Bawerk, 1949, p. 30).

${ }^{11}$ Ian Steedman declara que "dado que la tasa de ganancia y todos los precios de producción pueden determinarse sin referencia alguna a ninguna magnitud de valor, el 'problema de la transformación' es un problema falso, una quimera, no hay ningún problema consistente en derivar las ganancias de la plusvalía y los precios de producción que deben encontrarse" (Steedman, 1985, pp. 11-12).

${ }^{12}$ En "L'opera postuma di Carlo Marx", de 1895, Achille Loria señala: "Ningún economista con un poco de sentido común se ocupa, ni se ocuparía nunca, de un valor que no corresponde al de la venta de las mercancías y al que éstas jamás podrían venderse, porque un valor así de ajeno e invariable sería una especie de noúmeno, que nunca podría manifestarse y sobre el cual el teórico podría afirmar impunemente cualquier cosa. Ahora Marx, al sostener que el valor de las mercancías está determinado por el trabajo, pero que las mercancías no se venden conforme a su valor, llega precisamente a la creación absurda de un valor-noúmeno ajeno a la realidad y a las leyes mismas del pensamiento" (citado en Dostaler, 1980, pp. 90-91). Entre los autores marxistas contemporáneos que sostienen implícitamente la no necesidad de los valores para la determinación de los precios de producción se encuentran Duménil y Lévy, quienes consideran que "el problema de la transformación no es un problema de la derivación de los precios de producción a partir de los valores. El conocimiento de los valores no ayuda en la computación de los precios de producción. Realmente, la relación entre valores y precios es completamente independiente del hecho de que las tasas de ganancia sean igualadas" (Duménil y Lévy, 1993, p. 48).

${ }^{13}$ Se trata de interpretaciones que sostienen que los precios de producción son simples reguladores de los precios de mercado, los cuales son propuestos como únicos precios reales. Por otra parte, están las interpretaciones dualistas que sostienen que los valores y los precios constituyen dos sistemas distintos e independientes de determinación. También deben ser mencionadas las posiciones que colocan el dinero, en cuanto forma de valor, en el centro; dentro de ellas se ubican las denominadas temporal single-system y la new interpretation o single system labour theory of value.
} 
problema de la reducción de los trabajos concretos heterogéneos a trabajo social abstracto.

Este problema es identificado por Adam Smith al señalar que "aunque el trabajo es la medida real del valor de cambio de todos los bienes, generalmente no es la medida por la cual se estima ese valor. Con frecuencia es difícil averiguar la relación proporcional que existe entre cantidades diferentes de trabajo" (Smith, 1958, p. 32). Por su parte, Marx no ofrece, como veremos, una solución definitiva a esta cuestión, reduciéndola a un supuesto simplificador: considerar toda clase de fuerza de trabajo como fuerza de trabajo simple. El análisis de los diversos intentos para responder a la reducción supera los fines del presente texto; ${ }^{14}$ el objetivo de éste consiste en demostrar, siguiendo la lógica dialéctica, que dicha reducción a tiempo de trabajo social abstracto, en contra de la lectura marxista tradicional, sólo se logra plenamente en el momento en que los precios de producción se determinan en el intercambio. En otras palabras, los valores sociales definitivos de las mercancías y, por tanto, los tiempos de trabajo social abstracto se determinan recíproca y simultáneamente por mediación de los precios de producción, lo cual ocurre en el momento del intercambio. De ello se sigue que ambos problemas, el de la transformación y el de la reducción del trabajo, son dos procesos dialécticamente relacionados entre sí. Para mostrar este punto proponemos una reconstrucción del concepto de capital planteado por Marx.

Esta reconstrucción implica, así, que sólo hasta que este último momento es puesto, las categorías inmediatamente puestas en el momento que constituye el punto de partida de la presentación son plenamente fundamentadas. Esta reconstrucción se desarrollará en cuatro momentos de la estructura lógica de $E l$ capital: 1) el punto de partida (sección primera del tomo I), 2) el pasaje al capital en general (segunda sección del tomo I), 3) el pasaje a la aparición inmediata del capital en general (sección primera del tomo III) y 4) el pasaje a los muchos capitales productivos (segunda sección del tomo III).

\section{Primer momento: el PUNto de Partida}

El primer momento de la presentación corresponde a la apariencia inmediata de la circulación del capital, es decir, la circulación mercantil simple (M-D.D-M), donde las determinaciones y relaciones que corresponden a las formas inmediatas

\footnotetext{
${ }^{14}$ Para un comentario sobre las diferentes posiciones alrededor de la reducción del trabajo, véase la introducción de Robles (2011).
} 
de existencia mercantil del capital, es decir, la mercancía y el dinero, son puestas. ${ }^{15}$ El punto de partida es la forma de mercancía -con sus dos determinaciones fenoménicas inmediatas: valor de uso y valor de cambio- en cuanto la entidad más simple del capital, es decir, la "forma celular" de la riqueza económica capitalista. De los valores de cambio de las mercancías tales como se revelan inmediatamente en la circulación, es decir, como diferentes proporciones cuantitativas en que, como valores de uso cualitativamente diferentes, las mercancías se intercambian entre sí, Marx deriva los fundamentos de la circulación mercantil simple a través de un doble movimiento inverso que se refiere a la relación dialéctica esencia/apariencia y contenido/forma. En el primer movimiento, se derivan el valor en cuanto "la expresión más abstracta del capital mismo y de la producción fundada en éste" (Marx, 1982b, p. 315) ${ }^{16}$ y el trabajo abstracto como la sustancia del valor.

En cuanto una sustancia común cristalizada en las mercancías, el valor aparece aquí como lo que, en general, permitirá a las mercancías identificarse como iguales entre sí, a pesar de sus diferencias cualitativas como valores de uso, y la cantidad de aquél, medido en términos de tiempo de trabajo abstracto, lo que les permitirá ser equivalentes e intercambiarse en una determinada proporción cuantitativa. En el movimiento inverso, aquel que presenta la forma de valor, se deriva el dinero en cuanto la forma inmediata de existencia y medida social de los valores de las mercancías y, por tanto, su forma-precio más simple y general.

El primer movimiento se enfoca así en el pasaje de la forma inmediata de aparición de los valores de cambio de las mercancías a sus valores en cuanto el contenido común que los fundamenta. En la medida en que el valor representa (tiempo de) trabajo objetivado en las mercancías, este pasaje es, al mismo tiempo, un movimiento de abstracción hacia su sustancia: el trabajo abstracto. ${ }^{17} \mathrm{Se}-$

${ }^{15}$ Se puede decir que, en cuanto el sistema económico capitalista es un sistema mercantil, este momento corresponde a la posición de las determinaciones y las relaciones de la producción y la circulación mercantil en general, determinaciones que, sin embargo, serán negadas (no suprimidas) y, por lo tanto, puestas en la forma social capitalista cuando se pase al momento de la producción y la circulación del capital: "La forma que adopta la circulación cuando el dinero sale del capullo, convertido en capital, contradice todas las leyes analizadas anteriormente sobre la naturaleza de la mercancía, del valor, del dinero y de la circulación misma" (Marx, 1982a, p. 190).

${ }^{16} \mathrm{O}$, como lo define Dussel en términos hegelianos, "[el] ser del capital" (Dussel, 2005, p. 219).

${ }^{17}$ De acuerdo con la presentación de Marx, este movimiento de abstracción presupone la abstracción del valor de uso de las mercancías y, en consecuencia, del carácter útil del trabajo; véase Marx (1982a, pp. 89-90). Sin embargo, este movimiento de abstracción del trabajo no debe considerarse como inmediato o suficiente en cuanto que la separación del trabajo concreto no da como 
gún Marx, este movimiento de abstracción del trabajo es un proceso real que implica las reducciones cualitativa y cuantitativa del trabajo en forma simultánea. ${ }^{18}$ El lado de la calidad concierne a la reducción de todos los trabajos individuales gastados privadamente en la producción a trabajo social y a trabajo simple $^{19}$-lo que implica considerar el trabajo simple y el trabajo complejo (incluido el intensivo $)^{20}$ como dos trabajos cualitativamente diferentes cuya relación permite establecer una relación cuantitativa, es decir, el trabajo complejo como una potencia de trabajo simple-.$^{21}$ El lado de la cantidad concierne a la reducción de todos los tiempos de trabajo a cuantos ${ }^{22}$ de tiempo de trabajo simple socialmente medido. ${ }^{23}$

resultado directo trabajo abstracto. Ésta ha sido la interpretación del marxismo tradicional, que intenta reducir a la producción el proceso de abstracción y ha negado la importancia del intercambio como el momento lógico en que el trabajo objetivado privadamente puede devenir trabajo social abstracto. El error consiste en intentar derivar la determinación abstracta a partir de los elementos concretos y, por tanto, postular lo abstracto como inferior a lo concreto. En realidad, la abstracción no debe considerarse solamente como un poner de lado, de separar, la materia sensible, sino más bien constituye la superación y la reducción de ésta, considerada como lo concreto aparente, a lo esencial, o dicho en otras palabras, el movimiento de abstracción es el modo de superar lo concreto aparente de las cosas para reducirlas a su esencia.

18 "Con el objetivo de medir los valores de cambio de las mercancías según el tiempo de trabajo contenido en ellas, es menester reducir los propios y diversos trabajos a trabajo indiferenciado, uniforme, simple, en suma a trabajo cualitativamente igual, y que por ende sólo se diferencia cuantitativamente. Esta reducción aparece como una abstracción, pero es una abstracción que se lleva a cabo a diario en el proceso de la producción social. La reducción de todas las mercancías a tiempo de trabajo no es una abstracción mayor, pero a la vez no es una abstracción menos real que la reducción de todos los cuerpos orgánicos a aire" (Marx, 1980a, pp. 12-13). Véase, también, Marx, 1980c, p. 120.

19 "Ante todo, la simplicidad indiferenciada del trabajo es la igualdad de los trabajos de diferentes individuos, la relación recíproca de sus trabajos entre sí como tratándose de trabajos iguales, cosa que ocurre mediante la reducción efectiva de todos los trabajos a trabajo de la misma índole" (Marx, 1980a, p. 14); énfasis agregado.

20 "El trabajo es cualitativamente distinto, también, no sólo según las diversas ramas de la producción, sino según su mayor o menor intensidad, etcétera” (Marx, 1982b, p. 415); énfasis agregado.

21 "Se considera que el trabajo más complejo es igual sólo a trabajo simple potenciado, o más bien multiplicado, de suerte que una pequeña cantidad de trabajo complejo equivale a una cantidad mayor de trabajo simple" (Marx, 1982a, p. 54-55).

${ }^{22}$ Este término se usa no con el significado, más conocido hoy, que le da la física moderna, sino en su sentido hegeliano: "La cantidad, puesta esencialmente con la determinación exclusiva que en ella está contenida, es el cuanto, la cantidad limitada" (Hegel, 1997, pp. 79).

23 "Es sólo la cantidad de trabajo socialmente necesario [o el] tiempo de trabajo socialmente necesario para la producción de un valor de uso, lo que determina su magnitud de valor [...] 'En cuanto valores, todas las mercancías son, únicamente, determinada medida de tiempo de trabajo solidificado'" (Marx, 1982a, pp. 48-49). 
Sin embargo, a pesar de la importancia dada por Marx a la reducción del trabajo para la determinación del valor de las mercancías, la limita a un supuesto simplificador: "Para simplificar, en lo sucesivo consideraremos directamente toda clase de trabajo como fuerza de trabajo simple, no ahorrándonos con ello más que la molestia de la reducción" (Marx, 1982a, p. 55). Como veremos, este supuesto simplificador es sólo la primera manifestación de una serie de problemas que surgen en la presentación que hace Marx, a lo largo de El capital, de la determinación de los valores sociales de las mercancías como productos del capital.

El primer problema surge en referencia al contexto donde se realiza el proceso de la reducción. Lo expuesto por Marx al respecto ha dado lugar al menos a dos interpretaciones opuestas: una considera que todos los trabajos se ponen inmediatamente como trabajo social abstracto en el momento en que se objetivan en la producción de las mercancías, suponiendo con esto que la reducción no es necesaria, o que se realiza de alguna manera independiente de la conexión social de las mercancías en la esfera del intercambio y sin que tomen la forma dineraria; mientras que la segunda considera que la reducción de los trabajos sólo puede realizarse, como Marx lo señala en la Contribución, "violentamente" (forcibly) (Marx, 1970, pp. 59-60; 1980, pp. 44-45) a través de las relaciones de intercambio de las mercancías en que están objetivados en el proceso de intercambio y, por lo tanto, por mediación de la forma dineraria que ellas necesariamente adquieren allí. En cuanto que esta última interpretación es, desde nuestro punto de vista, la correcta, nos basaremos en ella en lo que sigue. ${ }^{24}$

El siguiente problema se refiere al carácter del trabajo abstracto: ¿Es fisiológico o social? La respuesta a esta pregunta es contradictoria (en sentido dialéctico), ${ }^{25}$ puesto que es realmente ambos. Esto es, en cuanto determinación natural de cualquier actividad individual de trabajo vivo, el carácter del trabajo

\footnotetext{
${ }^{24}$ Para un desarrollo amplio al respecto, véase Robles (2011).

${ }^{25}$ A diferencia de la lógica formal que intenta eliminar las contradicciones para poder arribar a la verdad -el principio de no contradicción y del tercero excluido son determinantes en este intento-, la dialéctica hegeliana sostiene que cualquier cosa finita aislada encierra una contradicción consigo misma pues requiere de otras formas de existencia para definir su propia existencia; en términos lógicos, puede establecerse dialécticamente un juicio de inclusión o incluyente (De Gortari, 1970) según el cual algo es $x$, o es $y$, o es ambos a la vez. Así, la contradicción no significa una ruptura en el desarrollo del pensamiento y la aproximación a la verdad, por el contrario, es el desarrollo de dichas contradicciones lo que permite el movimiento de la realidad. Cada elemento tiene el impulso para superar la contradicción y el resultado (o solución de la contradicción) involucra un movimiento hacia un elemento nuevo más complejo que elimina la contradicción original y que está intrínsecamente relacionado con el primer elemento. Este nuevo elemento tiene, de
} 
que se realiza inmediatamente en la esfera de la producción sólo puede ser un gasto de trabajo en sentido fisiológico, ${ }^{26}$ que, una vez que se ha objetivado en las mercancías, deviene una abstracción objetiva. Sin embargo, ésta es una abstracción objetiva que no ha sido todavía puesta en forma social. Esto implica que, para Marx, no es la realidad fisiológica del trabajo la que constituye el trabajo social abstracto, sino que éste sólo resulta de la posición social de esta realidad natural del trabajo y, por lo tanto, de su negación. ${ }^{27}$ De esta manera, el trabajo fisiológico abstracto sólo puede constituir la presuposición natural del trabajo social abstracto, el cual sólo puede ser puesto socialmente por mediación del intercambio de las mercancías en que está objetivado. Además, se agrega el hecho de que los trabajos individuales en sentido fisiológico no pueden constituir trabajos sociales abstractos porque, como tales, les falta el momento de la singularidad en cuanto una unidad social, la cual sólo se puede lograr, a través de la mediación del proceso social de intercambio de las mercancías, por la forma dineraria. ${ }^{28}$

igual manera, una nueva contradicción, lo que permite la continuidad del movimiento. En otras palabras, negar un elemento significa superarlo conservándolo.

26 "Todo trabajo es, por un lado, gasto de fuerza humana de trabajo en sentido fisiológico, y es en esta condición de trabajo humano igual, o de trabajo abstractamente humano, como constituye el valor de la mercancía. Todo trabajo, por otra parte, es gasto de fuerza humana de trabajo en una forma particular y orientada a un fin, y en esta condición de trabajo útil concreto produce valores de uso" (Marx, 1982a, p. 57); énfasis agregado. "El carácter místico de la mercancía no deriva, por tanto de su valor de uso. Tampoco proviene del contenido de las determinaciones de valor. En primer término, porque por diferentes que sean los trabajos útiles o actividades productivas, constituye una verdad, desde el punto de vista fisiológico, que se trata de funciones del organismo humano y que todas esas funciones, sean cuales fueren su contenido y su forma, son en esencia gasto de cerebro, nervio, músculo, órgano sensorio, etc., humanos" (Marx, 1982a, p. 87).

${ }^{27}$ Entender el trabajo social abstracto directamente como trabajo fisiológico es reducir la conceptualización del trabajo que constituye la sustancia del valor de Marx a una sustancia puramente físico-natural; lo que implica remitirla, como lo hace la economía política clásica y una parte importante de las tradiciones neorricardiana (sraffiana) y marxista, a una conceptualización que pertenece únicamente al universo antropológico general. De aquí que al trabajo abstracto se le conciba simplemente como una encarnación o incorporación (embodiment) real. En este sentido, el trabajo social abstracto tampoco puede ser considerado trabajo en general, sea en un sentido natural, es decir, como la generalización de las características fisiológicas comunes a todos los trabajos, ni tampoco una construcción subjetiva, es decir, como una simple generalización mental de todos los trabajos en cuanto trabajos concretos. Para una crítica a este respecto, véase Murray (2005) y Robles (2001).

${ }^{28}$ Según Marx, es esta unidad lo que quita la condición de sujetos a sus agentes individuales: "El trabajo, medido de esta suerte por el tiempo, no aparece de hecho como el trabajo de diversos sujetos, sino que los diferentes individuos que trabajan aparecen, antes bien, como meros órganos del trabajo [...] Es el tiempo de trabajo del individuo, su tiempo de trabajo, pero sólo en cuanto 
En consecuencia, en cuanto tiempos de trabajo fisiológico objetivado en la producción privada de las mercancías, sus valores sólo pueden constituir la presuposición natural de, o la determinación natural inmediata de, sus valores sociales y, como tales, debe considerarse la objetivación de ciertos montos de tiempo de trabajo fisiológico que fueron necesarios para producirlas privadamente. Como valores presupuestos, no puestos todavía como valores sociales, éstos pueden ser denominados valores individuales. Para citar a Marx:

El tiempo de trabajo social sólo existe, por así decirlo, en forma latente en [las] mercancías, y sólo se revela durante su proceso de intercambio. No se parte de los trabajos de los individuos en calidad de trabajo [social], sino a la inversa, de trabajos particulares de individuos privados, los cuales sólo en el proceso de intercambio, y por [la superación $]^{29}$ de su carácter originario, se revelan como trabajo social general. De ahí que el trabajo social general no sea una premisa acabada, sino un resultado en devenir (Marx, 1980a, p. 29). ${ }^{30}$

Lo que Marx deriva en el primer movimiento es así el "carácter originario" de la abstracción del trabajo objetivado en las mercancías, es decir, su determinación fisiológica inmediata, $\mathrm{y}$, por tanto, sus valores individuales, los cuales sólo podrán devenir (tiempos de) trabajo social abstracto objetivados en ellas por medio de su "superación" en el proceso de intercambio. Es decir, es sólo a través de la mediación del intercambio que los trabajos fisiológicos objetivados privadamente en la producción de las mercancías devienen en trabajo social y, entonces, en valores sociales. Es en este sentido que Marx dice que "ni un sólo átomo

tiempo de trabajo en común a todos, para lo cual resulta indiferente, por lo tanto, saber de cuál individuo es dicho tiempo de trabajo [...] El trabajo del individuo, para dar por resultado un valor de cambio, debe dar por resultado un equivalente general, es decir, la representación del tiempo de trabajo del individuo como tiempo de trabajo general o la representación del tiempo de trabajo general como el del individuo" (Marx, 1980a, pp. 13-15).

${ }^{29}$ En la traducción al español de Siglo XXI, esta palabra aparece como "supresión", mientras que en la edición en inglés traducida por S. W. Ryazanskaya aparece como "supersession" (Marx, 1970, p. 45), que en español puede ser traducida por "superación", con el significado no de supresión, sino de negación dialéctica. De acuerdo a la dialéctica de Marx, esta última traducción es la que parece ser correcta.

30 "El trabajo que se manifiesta en el valor de cambio se halla presupuesto como trabajo del individuo aislado. Este trabajo se torna social por el hecho de que asume la forma abstracta de su contrario directo, la forma del carácter general abstracto" (Marx, 1980a, p. 16). Véase, también, Marx (1982a, p. 89). 
de sustancia natural forma parte de [la] objetivación [de las mercancías] en cuanto valores [sociales]" (Marx, 1982a, p. 58).

El movimiento inverso corresponde al pasaje del valor individual de las mercancías a su forma-valor o valor de cambio socialmente determinado. Este movimiento es tratado por Marx como una unidad dialéctica de contenido y forma, en la cual ésta es considerada no sólo como fundamentada por aquél, sino también como parte de la determinación del contenido. ${ }^{31}$ Dado que el valor individual es un contenido esencial que no puede aparecer directamente en el valor de uso de la mercancía que lo porta, éste tendrá necesariamente que expresarse en el valor de uso de una mercancía diferente, mostrando así la importancia de la forma aparente para el contenido esencial. ${ }^{32}$ Tras el desarrollo de la forma del valor, la mercancía específica en cuyo valor de uso los valores individuales de todas las mercancías se expresan es la que toma la forma dineraria de las mercancías. ${ }^{33}$ Como expresión material de los valores individuales de las mercancías, el dinero deviene el "ser-alli" de sus valores, es decir, la forma inmediata de existencia social de sus valores individuales ${ }^{34} \mathrm{y}$, consecuentemente, de los tiempos de trabajo fisiológico que los representan como tiempos de trabajo social abstracto. ${ }^{35}$

${ }^{31}$ En este sentido, en la Ciencia de la lógica se lee: "La forma, ante todo, está frente a la esencia; así es relación fundamental en general y sus determinaciones son el fundamento y lo fundado. Después está frente a la materia; aquí es reflexión que determina y sus determinaciones son la determinación reflexiva misma y su subsistir. Finalmente, está frente al contenido; y de este modo sus determinaciones son de nuevo la forma misma y la materia. Lo que anteriormente era lo idéntico consigo mismo, vale decir, en primer lugar el fundamento, después el subsistir en general y por último la materia, cae bajo el dominio de la forma y es de nuevo una de sus determinaciones" (Hegel, 1968, pp. 400-401).

${ }^{32}$ Esto se refiere al desdoblamiento de la mercancía en dinero o la génesis del dinero en Marx. Para un tratamiento dialéctico al respecto, véase el capítulo 2 de Meany (2002) y el capítulo 5 de Robles (2005).

${ }^{33}$ Con la posición de la forma dineraria, uno pasa de la situación en la cual la materia (es decir, el valor de uso) era el portador de la forma (es decir, el valor individual) a la situación en la que la forma-valor se encarna en la materia (es decir, el valor de uso de la mercancía específica que funciona como dinero).

${ }^{34}$ De acuerdo con Hegel, se podría decir que en la medida que el valor, en cuanto ser del capital, no sea determinado socialmente, no es nada.

${ }^{35}$ La mercancía dineraria es así un universal que, al mismo tiempo, es un singular, es decir, un universal concreto. Dice en el apéndice "La mercancía" de El capital: "Es como si además y aparte de los leones, tigres, liebres y de todos los restantes animales reales, que agrupados conforman los diversos géneros, especies, subespecies, familias, etcétera, del reino animal, existiera también el animal, la encarnación individual de todo el reino animal. Tal individuo, que en sí mismo engloba todas las especies efectivamente existentes de la misma cosa, en un ente general, como animal, Dios, etc." (Marx, 1984c, p. 998). 
"Como valor -dice Marx- ella [la mercancía] es dinero" (Marx, 1984a, p. 65). De aquí que sólo por medio de la forma dineraria que las mercancías adquieren en el intercambio, sus valores individuales y el tiempo de trabajo fisiológico que los representan se ponen como las formas inmediatas en que existen socialmente:

Esta necesidad de que el trabajo individual se represente como trabajo general es la necesidad de la representación de la mercancía como dinero [...]. Por eso, en cuanto a la existencia de la mercancía como dinero no sólo hay que destacar que las mercancías se dan en el dinero una determinada medida de sus magnitudes de valor -en cuanto expresan todo su valor en el valor de uso de la misma mercancía-, sino, además, que se representan todas como existencia del trabajo social, general abstracto; una forma en las que todas poseen la misma forma; todas aparecen como encarnación del trabajo social y, en cuanto tal, todas ellas, como efecto de la existencia del trabajo social, son directamente cambiables en proporción a su magnitud de valor- por todas las otras mercancías (Marx, 1980b, p. 120).

Tal necesidad se presenta así como una transformación cualitativa que toma la forma de una relación cuantitativa, esto es, en cuanto unidad de calidad y cantidad, el dinero deviene la medida social de los valores individuales de las mercancías como valores sociales. En otras palabras, en la medida en que es por mediación del dinero que las cantidades de tiempo de trabajo fisiológico devienen cuantos de tiempo de trabajo social abstracto, es por su mediación que los valores sociales de las mercancías son cualitativa y cuantitativamente puestos. La posición tanto cualitativa como cuantitativa del valor social de las mercancías está así unificada en su forma inmediata de existencia social, es decir, su forma dineraria.

\section{Valor social y la forma-precio más general}

Una vez que se ha establecido la unidad de medida de la mercancía dineraria, la expresión del valor de las mercancías en una cantidad determinada de esta unidad deviene su forma dineraria o forma-precio: por ejemplo, una "ecuación aislada, como 1 tonelada de hierro $=2$ onzas de oro, basta ahora para representar [idealmente] el valor [individual] del hierro de una manera dotada de vigencia social" (Marx, 1982a, p. 116) (énfasis agregado). Ésta es la forma-precio más simple y general del valor social de cualquier mercancía. A este nivel de la pre- 
sentación, el valor de la mercancía es fundamental "porque -como dice Marx-el dinero sólo puede desarrollarse conceptualmente a partir de este fundamento, y porque el precio, con arreglo a su concepto general, sólo es, en primera instancia, el valor en forma dineraria" (Marx, 1976, p. 244). El cuadro 1 expresa la determinación del valor social de una mercancía, $C_{i}$, por mediación de su forma dineraria o forma-precio más simple y general:

Cuadro 1. Representación gráfica de la determinación del valor social de una mercancía por mediación de su forma-precio más simple y general

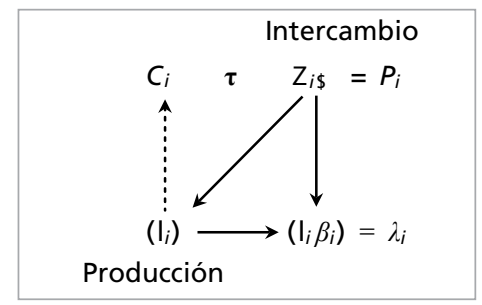

Donde: $\mathrm{I}_{i} \quad=$ un determinado monto de tiempo de trabajo fisiológico.

.... = la objetivación inmediata del tiempo de trabajo fisiológico en la producción de $C_{i}$;

$\tau=$ la relación de equiparación monetaria de la mercancía o la expresión del valor individual de $C_{i}$ en el dinero;

$Z_{i \$}=$ las unidades monetarias que representan la forma-precio de $C_{i}$;

$P_{i} \quad=$ la forma-precio de $C_{i}$;

$\longrightarrow=$ las relaciones de determinación del valor social de $C_{i}$ por mediación de su forma dineraria;

$\beta_{i}=$ el coeficiente de reducción, que expresa la transformación de unidades de tiempo de trabajo fisiológico, $\mathrm{I}_{i}$, en unidades de tiempo de trabajo social, $1_{i} \beta_{i}$

$\lambda_{i} \quad=\mathrm{I}_{i} \beta_{i}=$ el valor social de $C_{i}$.

En dicho cuadro se muestra que, en general, el valor individual de una mercancía, es decir, una cierta cantidad de tiempo de trabajo fisiológico, $1_{i}$, objetivado directamente en ella, se pone como valor social, $\lambda_{i}$, es decir, como un cuanto de tiempo de trabajo social abstracto $\left(1_{i} \beta_{i}\right)$, por mediación de su formaprecio en el intercambio. Esta transformación se expresa por el coeficiente de reducción $\beta_{i}$, que representa la posición cualitativa y cuantitativa del trabajo abstracto fisiológico como trabajo abstracto socialmente medido por mediación del dinero. Esta transformación representa así el pasaje del espacio del tiempo de trabajo fisiológico al espacio del tiempo de trabajo social abstracto por mediación de su forma de existencia socialmente determinada: la forma dineraria. 
Sin embargo, aunque en la circulación mercantil simple, M-D.D-M, el valor de las mercancías adquiere una forma general de existencia social y autónoma en el dinero, éste no se presenta como un valor que se mantiene y reproduce a sí mismo, es decir, como un valor en proceso. Esto es evidente en que la finalidad de este proceso no es la valorización del valor, sino el valor de uso de las mercancías, que las mercancías no se presentan como productos del capital, sino como simples mercancías y que, por lo tanto, este proceso no contenga dentro de sí el principio de autorenovación necesaria para sostenerse por sí mismo. Estas insuficiencias bastan para hacer necesario el pasaje al siguiente momento de la presentación.

\section{Segundo momento: el Pasaje al CAPITAl EN General}

El segundo momento corresponde a la conversión, o al proceso del devenir, de la forma dineraria del valor en capital en general, es decir, el concepto de capital tal como es esencialmente determinado. Primeramente, Marx presenta este movimiento como una unidad de tres determinaciones que pertenecen al proceso de la circulación: a) la determinación cualitativa de su devenir: D-M-D, b) la determinación cuantitativa de su devenir: D-M-D' y c) la medida en cuanto unidad de sus determinaciones cualitativas y cuantitativa. ${ }^{36}$

La determinación cualitativa se refiere al movimiento cíclico D-M-D, cuyo objetivo es el dinero como un fin en sí mismo, mediante el cual el valor que originalmente es adelantado en forma dineraria atraviesa sucesivamente las fases antitéticas D-M y M-D, en las que se pone a sí mismo en las formas de dinero (D) y mercancía (M), que asume y toma en turno sin que se pierda dentro y a través de este movimiento. Como una unidad continua de dinero y mercancía, el valor, dice Marx, "es capital, y ese propio ponerse se presenta como la circulación del capital" (Marx, 1984a, p. 206). El valor, que inicialmente se reveló como un predicado del dinero y de la mercancía y, por tanto, en cierta medida como una sustancia inerte en la circulación mercantil simple M-D.D-M, se pone a través de este movimiento como el sujeto esencial de la circulación de capital, es decir, el ser esencial del capital como una sustancia con movimiento propio, y el dinero y las mercancías devienen sus formas materiales de existencia como capital. Así, el dinero como dinero es negado y superado como una cosa material tangible, con-

${ }^{36}$ A este respecto, véase la sección 2.3 de Robles (2008) y el capítulo 1, sección 1.2, de Robles (2011). 
virtiéndose en un proceso. Sin embargo, esta determinación es insuficiente para explicar por completo la conversión del valor en capital, debido al hecho de que el resultado "en el que se consuma todo ese proceso es el intercambio de dinero por dinero, D-D..., lo mismo por lo mismo", que "parece ser una operación tan carente de objetivos como absurda" (Marx, 1982a, pp. 180 y 183).

La superación de la insuficiencia de la determinación cualitativa nos remite a la determinación cuantitativa, es decir, la finalidad y resultado de este movimiento tiene que referirse a la diferencia cuantitativa con respecto a la cantidad de la forma-valor originalmente adelantada, lo que implica ir más allá de su propio límite cuantitativo. Para Marx, el valor que no sólo se mantiene y perpetúa a sí mismo a través de la circulación (su determinación cualitativa), sino que además aumenta su propia magnitud agregando a sí mismo un plusvalor, se convierte en capital como valor que se valoriza a sí mismo, que se automultiplica.

Es por esto que, según Marx, "la forma plena de este proceso es [...] DM-D', donde $\mathrm{D}^{\prime}=\mathrm{D}+\Delta \mathrm{D}$, esto es, igual a la suma de dinero adelantada más un incremento"] (Marx, 1982a, p. 184). Después de resumir esta conversión, Marx sostiene:

Pero más aún. En vez de representar relaciones mercantiles, aparece ahora, si puede decirse, en una relación privada consigo mismo. Como valor originario se distingue de sí mismo como plusvalor -tal como Dios Padre se distingue de sí mismo en cuanto Dios Hijo, aunque ambos son de una misma edad y en realidad constituyen una sola persona-, puesto que sólo en virtud del plusvalor de $£ 10$, las $£ 100$ adelantadas se transmutan en capital, y así que esto se efectúa, así que el Hijo es engendrado y a través de él el Padre, se desvanece de nuevo su diferencia y ambos son uno, £110 (Marx, 1982a, p. 189).

Dado que el devenir del valor en la cualidad de capital se debe al incremento cuantitativo de valor que él mismo crea de sí mismo a través de su propio proceso de circulación, la posición del valor como capital se manifiesta por la relación recíproca de los cuantos relacionados de valor incorporados en su propio resultado, es decir, el devenir de ambos, el cuanto de valor originalmente adelantada (D: el Padre) y el cuanto de plusvalor que se relaciona con él como su propio incremento ( $\Delta \mathrm{D}$ : el Hijo), en capital (D': el Dios Único). El Padre crea al Hijo, así como el Hijo crea al Padre. Esta posición cualitativa de los cuantos relacionadas de valor, es decir, de las partes constitutivas del valor que se ha 
valorizado a sí mismo, se refiere a la propia medida de realización del valor como capital.

Conforme a la naturaleza de éste, primeramente en el capital -al haberse transformado en dinero- sólo se presenta medido el nuevo valor, creado por el capital; es decir, se reproduce la primera determinación del dinero como la medida general de las mercancías; ahora como medida de la plusvalía, de la valorización del capital. Bajo la forma de dinero, este valorizarse se presenta como medido en sí mismo, como teniendo en sí mismo su medida (Marx, 1984a, p. 408) (énfasis agregado). ${ }^{37}$

La relación cualitativa a través de la cual el devenir del capital se mide a sí mismo puede ser así representada por la razón cuantitativa que denota el cuanto de plusvalor $(\Delta \mathrm{D})$ que una determinada cantidad de valor dinerario como capital adelantado (D) ha creado en un periodo determinado de tiempo: esto es, $\Delta \mathrm{D} / \mathrm{D}$. En cuanto que representa la posición del valor como capital, ésta puede definirse como la tasa de valorización del capital en general. Esta tasa es la medida del capital, es decir, la medida de su valorización. Dicha tasa es así la presuposición lógica de la tasa de ganancia del capital, la cual sólo será puesta en el momento en que el capital se presente como apariencia, es decir, hasta el tercer tomo de $E l$ capital.

Sin embargo, la consideración de que las metamorfosis que sufre el valor durante el proceso de circulación, D-M-D', se llevan a cabo, al igual que en el proceso de la circulación mercantil simple, M-D.D-M, bajo la ley del intercambio de equivalentes,${ }^{38}$ tiene la implicación de que este proceso por sí mismo no puede explicar el origen de la valorización, es decir, la creación del plusvalor. Es por esto que, aunque necesarias, las determinaciones del devenir del capital que pertenecen al proceso de circulación son insuficientes para fundamentar por completo el devenir de valor dinerario originalmente adelantado en capital. La

${ }^{37}$ En el tomo II de El capital, lo dice de la siguiente manera: "Pero D' como D $+d$, es decir, $£$ 500 como $£ 422$ de capital adelantado más un incremento del mismo de $£ 78$, representa al mismo tiempo una relación cualitativa, aunque esta misma relación cualitativa sólo existe como relación entre las partes de una suma homogénea, es decir, como relación cuantitativa" (Marx, 1978, p. 53) (énfasis agregado).

38 "La transformación del dinero en capital ha de desarrollarse sobre la base de las leyes inmanentes al intercambio de mercancías, de tal modo que el intercambio de equivalentes sirva de punto de partida" (Marx, 1982a, p. 2002). 
dificultad para superar estas insuficiencias es que "el capital [...] no puede surgir de la circulación, y es igualmente imposible que no surja de la circulación" (Marx, 1982a, p. 202). Esta dificultad es resuelta por la introducción del trabajo, vía la compra de la fuerza de trabajo en la circulación, a "la oculta sede de la producción", donde, por medio de su consumo productivo junto con los medios de producción, el secreto de la producción de plusvalía sale a la luz, lo que permite, a su vez, llegar al corazón de la cuestión relativa a "no sólo cómo el capital produce, sino también cómo se produce el capital" (Marx, 1982a, p. 214).

Esto implica que, para convertirse realmente en capital, es de necesidad que éste se relacione de forma negativa con su propio otro, es decir, con el trabajo como no capital, pero que a través de su negación se convierte en capital. Cada uno de ellos es así la negación, o "no ser", del otro. El trabajo adquiere así una determinación más: trabajo que produce capital como su propio otro. Esta relación negativa del trabajo con el capital es la razón por la que son opuestos uno del otro ${ }^{39}$ y por la que también son opuestos sus respectivos portadores, los capitalistas y los trabajadores asalariados. Con esto, se pone lo que estaba presupuesto, es decir, el trabajo que estaba puesto como trabajo que producía valor y dinero en la circulación mercantil simple, ahora se pone como trabajo que produce capital. De esta manera, el capital puede entenderse como la sustancia abstracta valor que se transforma en sujeto, es decir, como un valor con movimiento propio, por medio del cual se valoriza a sí mismo.

Aunque la (re)producción del capital no será tratada aquí, tres puntos necesitan ser mencionados: primero, como productos del capital, el dinero y las mercancías devienen en sus propias formas de existencia puestas por él y, como tales, se convierten en las condiciones materiales de la reproducción del capital y de las clases sociales que componen la sociedad capitalista. ${ }^{40}$ Segundo, considerado como una unidad de sus propios procesos de producción y de circulación a través de los cuales se mantiene y multiplica a sí mismo, el capital se autopresenta como el sujeto de un creciente movimiento en forma de "una línea en espiral,

\footnotetext{
${ }^{39}$ Es en este sentido que Hegel afirma: "Porque cada uno de los dos en tanto es por sí en cuanto no es el otro. La diferencia de la esencia es, por tanto, la contraposición, según la cual lo diferente no tiene frente a sí un otro en general, sino su otro; esto es, cada uno tiene su propia determinación sólo en su relación con el otro; es reflejado en sí sólo en cuanto es reflejado en el otro, e igualmente el otro; cada uno es, de este modo, su otro del otro" (Hegel, 1997, p. 70; Hegel, 1974, p. 98).

${ }^{40}$ Véase Marx (1984b, p. 712). Cabe aclarar que, en la medida en que la reproducción se refiere al capital y no a meras relaciones técnicas, la existencia material de productos (como medios de producción e insumos) no es condición suficiente para reconstituir el proceso económico capitalista.
} 
una curva que se amplía, no un simple círculo" (Marx, 1984a, p. 206). ${ }^{41}$ Y tercero, como una consecuencia del movimiento de la reproducción del capital, "la ley de la apropiación o ley de la propiedad privada, ley que se funda en la producción y circulación de mercancías, se trastroca, obedeciendo a su dialéctica propia, interna e inevitable, en su contrario directo" (Marx, 1984b, pp. 720-721), es decir, en la ley de apropiación capitalista. Lo que esto significa es que el intercambio de equivalentes se transforma en la apropiación por parte del capital, sin equivalente, de la fracción no pagada del trabajo realizado por los asalariados. Dicha fracción no pagada, como se explica a continuación, forma parte del precio.

\section{Valor social y precio directo}

De acuerdo con Marx, los elementos constitutivos de la forma dineraria del valor de las mercancías como productos de capital son: 1) las partes constitutivas de la suma de valor dinerario originalmente adelantado como capital (D), en la compra de las mercancías (M), necesarias para su valorización en la producción, es decir, el capital constante (medios de producción) y el capital variable (fuerza de trabajo), y 2) el plusvalor como el tiempo de trabajo no remunerado extraído directamente a los trabajadores asalariados. La suma de dinero que equivale al valor valorizado (D'), objetivado en las mercancías producida como productos de capital (M'), está, por lo tanto, compuesta por el valor del dinero originalmente adelantado, $\mathrm{D}=c$ (capital constante $)+v$ (capital variable), más un plusvalor, $p v$. De aquí que, en general, la forma-precio del valor de cualquier mercancía como producto de capital pueda representarse como:

$$
P=c+v+p v
$$

Con la posición de esta forma-precio, la forma dineraria más simple y general de la mercancía es negada y superada, obteniendo así un mayor grado de concreción y determinación.

En el capítulo 10 del tomo i de El capital, Marx introduce el concepto de valor social de las mercancías del mismo tipo elaboradas por diferentes productores individuales en el contexto de una rama particular: "El valor real de una mercancía, sin embargo, no es su valor individual, sino su valor social, esto es, no se mide por el tiempo de trabajo que insume efectivamente al productor en

\footnotetext{
${ }^{41}$ Véase, también, Marx, 1982b, p. 131.
} 
cada caso individual, sino por el tiempo de trabajo requerido socialmente para su producción" (Marx, 1984b, p. 385). ${ }^{42}$

De los propios escritos de Marx, la determinación del valor social de las mercancías del mismo tipo se ha interpretado de dos maneras opuestas, que de hecho corresponden a sendas interpretaciones acerca de la reducción del trabajo que fueron discutidas en el primer momento. Una considera que esta determinación es el resultado de la media ponderada de la suma de todos los tiempos de trabajo individuales del conjunto de productores:

El valor total producido dividido entre el número de productos determina el valor de cada producto y sólo como tal parte alícuota se convierte en mercancía. Ya no es el trabajo empleado en cada mercancía particular [...], sino el trabajo total, una parte alícuota del cual, la media del valor total [dividido] entre el número de productos, determina el valor de cada uno de éstos y lo constituye en mercancía (Marx, 1980c, p. 98). ${ }^{43}$

Esta interpretación sólo puede ser válida si todos los tiempos de trabajo individuales objetivados directamente en la producción de las mercancías son inmediatamente considerados como cantidades de tiempo de trabajo social abstracto, lo que implicaría además que, independientemente de la diferencia en los métodos y condiciones de producción utilizada por los productores individuales, cualquier unidad individual de tiempo de trabajo gastada inmediatamente en la producción genera una misma unidad de valor social y que, dependiendo del método y de las condiciones de producción de cada uno de aquéllos, la determinación del valor social de las mercancías se traducirá en la realización de menos o más valor y, por lo tanto, de menos o más plusvalor que los producidos por cada uno. ${ }^{44}$ Además de esto, debe señalarse que una media ponderada es siempre el resultado de una construcción subjetiva.

42 "El valor de una mercancía, en efecto, se determina por la cantidad de trabajo contenido en ella, pero esa cantidad misma está determinada socialmente" (Marx, 1982a, p. 253).

${ }^{43}$ Véase también, Marx, 1980b, p. 185.

${ }^{44}$ Esto lo dice Marx explícitamente en un pasaje del tercer tomo de El capital, donde el valor social toma la forma de valor de mercado: "El valor individual de algunas de esas mercancías estará por debajo del valor de mercado (es decir, que se requerirá menos tiempo de trabajo para su producción de lo que expresa el valor de mercado); el de otras estará por encima de él. Por una parte habrá que considerar al valor de mercado como el valor medio de las mercancías producidas en una esfera, mientras que por otra habrá que hacerlo como el valor individual de las mercancías que se producen bajo condiciones medias de esa esfera y que constituyen el grueso de los productos 
Por el contrario, la otra interpretación considera la determinación del valor social de las mercancías del mismo tipo como resultado de un proceso objetivo de reducción: "en todo proceso de formación de valor siempre es necesario reducir el trabajo calificado a trabajo social medio, por ejemplo, un día de trabajo calificado a $x$ día de trabajo simple" (Marx, 1982a, p. 240). Así, esta determinación puede ser entendida como el resultado de un proceso posterior de medición social a través del cual todos los tiempos de trabajo individuales en sentido fisiológico que están objetivados en la producción de la masa total de las mercancías del mismo tipo dentro de una rama, independientemente de sus condiciones y métodos de producción individuales, se ponen como cantidades de tiempo de trabajo abstracto socialmente medido, esto es, como un mismo valor social, por mediación de su mismo precio directo (o, dicho de otra manera precio proporcional al valor social).

Considerando que esta última interpretación es la correcta, el cuadro 2 muestra el proceso de determinación del valor social de las mercancías del mismo tipo, en los supuestos de que sólo hay dos productores, cada uno con un método diferente de producción, y que los medios de producción son capital constante circulante. Dicho cuadro muestra la posición (o transformación) de los valores individuales de dos mercancías, $C_{i}$, del mismo tipo elaboradas por dos diferentes productores individuales, valores que representan diferentes cantidades de tiempo de trabajo fisiológico, $1_{i}$, objetivado directamente en su producción, más el valor de los medios de producción, $\lambda_{M P}$, transferido a cada una de ellas, en un mismo valor social, $\lambda^{\circ}$, es decir, un determinado cuanto de tiempo de trabajo social $\left(\lambda_{M P i}+1_{i} \beta^{\circ}\right)$, por medio de su mismo precio directo, $P_{D}$, en la esfera del intercambio.

Así, el precio directo de las mercancías del mismo tipo producidas por cualquier productor individual puede representarse mediante la siguiente fórmula:

$$
P_{D}=c_{i}+\left(v_{i}+p v_{i}\right) \Rightarrow \lambda_{M P i}+\left(1_{i} \beta_{i}^{\circ}\right)
$$

donde $c_{i} \mathrm{y}\left(v_{i}+p v_{i}\right)$ son proporcionalmente iguales a $\lambda_{M P i}$ y $\left(l_{i} \beta_{i}^{\circ}\right)$, de manera respectiva.

de la misma [...] Cuando la oferta de las mercancías al valor medio, es decir al valor medio de la masa ubicada entre ambos extremos, satisface la demanda habitual, las mercancías cuyo valor individual se haya por debajo del valor de mercado realizan un plusvalor extraordinario o plusganancia, mientras que aquéllas cuyo valor individual se haya por encima del valor de mercado no pueden realizar una parte del plusvalor contenido en ellas" (Marx, 1976, p. 225-226). 
Cuadro 2. Representación gráfica de la determinación del valor social del mismo tipo de mercancías por mediación de su precio directo

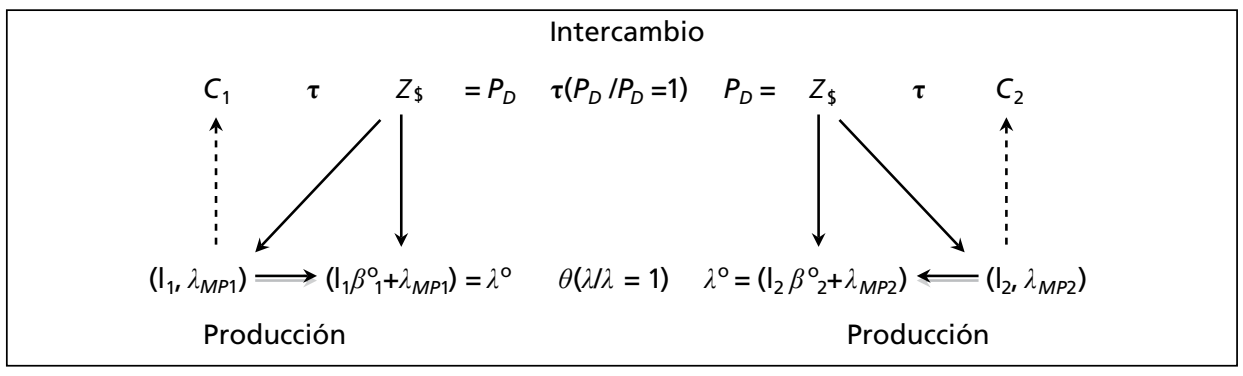

Donde: $\mathrm{I}_{i} \quad$ = tiempo de trabajo individual en sentido fisiológico gastado en la producción de $C_{i}$;

$\lambda_{M P i}=$ el valor social de, o el tiempo de trabajo social objetivado en, los medios de producción utilizados en la elaboración de $\mathrm{C}_{\mathrm{i}}$;

...- = la objetivación de los tiempos de trabajo directos e indirectos en la producción de las mercancías;

$\tau=$ la relación de igualación de las mercancías en el espacio monetario, representando la expresión del valor individual de las mercancías en el dinero;

$Z_{\mathrm{s}} \quad$ = las unidades monetarias que representan el precio directo de las mercancías;

$P_{D}=$ el precio directo de las mercancías;

$\longrightarrow=$ las relaciones de determinación del valor social de las mercancías por mediación de su precio directo;

$\beta^{\circ}{ }_{i}=$ los coeficientes de reducción que expresan la transformación de unidades de tiempo de trabajo en sentido fisiológico, $\mathrm{l}_{\mathrm{i}}$, en unidades de tiempo de trabajo social;

$\mathrm{I}_{i} \beta_{i}=$ el tiempo de trabajo social directo, o el valor agregado social, objetivado en $C_{i} ;$

$\lambda^{0}=$ el mismo valor social de las mercancías es cuanto el tiempo de trabajo social que representa su mismo precio directo;

$\theta \quad=$ la relación de igualdad de las mercancías en el espacio del valor social.

Sin embargo, además de reconocer que la reducción de todos los trabajos individuales es "inevitable", Marx la constriñe a una operación superflua: "Si suponemos [...] que el obrero empleado por el capital ejecuta un trabajo social medio simple, nos ahorramos una operación superflua y simplificamos el análisis" (Marx, 1982a, p. 240). Pero en este artículo se está en desacuerdo con tal juicio, porque, incluso para él mismo, la posición del valor social de las mercancías requiere que la reducción del trabajo deba realizarse inevitablemente no sólo para todos los trabajos individuales del mismo tipo que se gastan dentro de una misma rama de la producción, sino para todos los trabajos individuales de diferente tipo con que se elaboran las distintas mercancías en todas las ramas que constituyen la producción social en su conjunto: "Basta señalar que esa re- 
ducción se lleva a cabo de hecho, cuando se ponen como valores los productos de todos los tipos de trabajo. En cuanto valores son equivalentes según ciertas proporciones; los mismos tipos superiores de trabajo se evalúan en trabajo simple" (Marx, 1982b, p. 415) (énfasis agregado). Lo que resulta claro de esta última referencia es que la reducción del trabajo dentro de una rama de la producción es aún insuficiente para la posición de todos los tipos de trabajos como trabajos sociales actualizados y, por tanto, es también insuficiente para la posición de la forma valor de las mercancías producidas en todas las ramas como valores sociales definitivos o actualizados.

\section{Tercer Momento: el PASAJe A La APARIENCIA Del CAPITAL EN GENERAL}

Como en cualquier pasaje a un momento más concreto de la presentación de Marx, el del momento en que el capital en general se refleja en la apariencia implica una inversión dialéctica: "Pero en la realidad (es decir, en el mundo de los fenómenos), las cosas aparecen invertidas" (Marx, 1976, p. 54). En particular, lo que Marx presenta aquí son las implicaciones lógicas de la conversión del "plusvalor y la tasa de plusvalor" que "son, relativamente hablando, lo invisible y lo esencial", en "la tasa de ganancia, y por ende la forma de plusvalor en cuanto ganancia", que "se revelan en la superficie de los fenómenos" (Marx, 1976, p. 49).

El punto de partida en esto es la forma dineraria en la que aparecen los elementos constitutivos del valor de la mercancía como producto del capital: $M=p c+p v$, donde $p c$ representa el precio de costo, es decir, la parte del valor que sustituye el capital total adelantado en su producción, y $p v$, el plusvalor, que aparece como un simple exceso de valor respecto al precio de costo. Como tal exceso, el plusvalor no parece derivar del trabajo (no pagado) objetivado en la producción, sino de todas las partes del capital total adelantado. Dice Marx (1976, p. 40):

Como vástago así representado del capital global adelantado, el plusvalor asume la forma trasmutada de la ganancia. De ahí que una suma de valor es capital porque se la desembolsa para generar una ganancia, o bien la ganancia resulta porque se emplea una suma de valor como capital. Si denominamos $g$ a la ganancia, la fórmula $M=c+v+p v=p c+p v$, se convierte en esta otra: $M=p c+g$, o sea valor de la mercancía $=$ precio de costo + ganancia . 
En el momento en que el capital en general aparece en la superficie de los fenómenos, el plusvalor adquiere, es puesto en, la forma dineraria de ganancia. Sin embargo, como la forma de aparición del plusvalor, la ganancia debe ser esencialmente entendida no sólo como un exceso sobre el precio de costo que resulta simplemente del proceso de la circulación, sino como algo que ha sido puesto y fundamentado por el capital mismo, en cuanto sujeto del proceso, y, por lo tanto, como resultado del automovimiento del capital como una relación consigo mismo que se refleja en la apariencia.

En el plusvalor queda al descubierto la relación entre capital y trabajo, en la relación entre capital y ganancia, es decir, entre el capital y el plusvalor -tal como éste aparece, por una parte, como excedente por encima del precio de costo de la mercancía, realizado en el proceso de la circulación, y por la otra como excedente más exactamente determinado en virtud de su relación con el capital global-, se presenta el capital como relación consigo mismo, una relación en la cual se distingue como una suma originaria de valor, de un valor nuevo puesto por él mismo (Marx, 1976, pp . 55-56).

Como resultado de su relación con el capital total adelantado, el plusvalor, como ganancia debe medirse por el propio capital, es decir, a través de su relación consigo mismo como un valor que se autovaloriza. En la medida en que corresponde al momento de aparición del capital en general, esta medida se refiere a la tasa de ganancia del capital en general, la cual es determinada por la proporción del plusvalor en la forma de ganancia que ha creado el capital presupuesto (es decir, el capital total adelantado) en un periodo determinado de tiempo. Esta tasa expresa así la proporción en que el capital mismo ha aumentado su propio valor:

El capital, partiendo de sí mismo como del sujeto activo, del sujeto del proceso [...] se comporta consigo mismo como valor que se aumenta a sí mismo, esto es, se comporta con la plusvalía como puesta y fundada por él; se vincula como fuente de producción consigo mismo en cuanto producto; como valor productivo, consigo mismo en cuanto valor producido. Por ello el valor recién producido ya no lo mide por su medida real, la proporción entre el plustrabajo y el trabajo necesario, sino que lo mide por sí mismo, por el capital, como supuesto de ese valor. Un capital de un valor determinado produce en un lapso determinado una plusvalía determinada. La plusvalía medida así por el valor del capital 
presupuesto -y puesto así el capital como valor que se valoriza a sí mismo- es el beneficio; bajo este specie - no aeterni sino capitalis- la plusvalía es beneficio, y el capital en sí mismo como capital, como valor que produce y reproduce, se diferencia de sí mismo como beneficio, valor recién producido. El producto del capital es el beneficio. Por consiguiente la magnitud de la plusvalía es medida por la magnitud de valor del capital, y la tasa del beneficio está por lo tanto determinada por la proporción entre su valor y el valor del capital (Marx, 1982b, p. 278).

Así, en el momento en que el capital en general se refleja a sí mismo en la apariencia y, por tanto, en el momento en que su medida esencial (es decir, su tasa de valorización, como se estableció en el segundo momento) deviene en tasa general de ganancia del capital y el plusvalor como una "specie capitalis" deviene ganancia, ${ }^{45}$ es que éste se pone como capital en general existente.

\section{Valor social y la forma-precio general}

Si se considera que $p c$ representa el precio de costo y $r=g / K$ la tasa general de ganancia (donde $g$ representa ganancias y $K$ el capital total adelantado), la forma-precio general del valor de las mercancías como productos de capital, adquiere una forma más concreta:

$$
P=p c+r K
$$

Sin embargo, debido al hecho de que, como Marx afirma, el "capital existe y sólo puede existir como muchos capitales [y] por consiguiente su autodeterminación se presenta como acción recíproca de los mismos entre sí” (Marx, 1984a, p. 366), el concepto de capital en general, como una relación consigo mismo que se autorrrefleja en la apariencia, es insuficiente para que se ponga y actualice como un capital socialmente existente.

\section{Cuarto momento: el Pasaje a la multiplicidad del CAPITAL}

Este momento implica dos pasajes: a) del capital en general tal como se refleja a sí mismo en la apariencia a los muchos capitales y $b$ ) de los muchos capitales al

\footnotetext{
${ }^{45}$ O, como Marx lo dice en palabras hegelianas: "El excedente, cuando [...] se refleja en sí mismo a partir de la tasa de ganancia o, de otro modo, el excedente, caracterizado más exactamente por la tasa de ganancia, se presenta como un excedente que el capital produce en forma anual o en un período de circulación determinado, más allá de su propio valor” (Marx, 1976, p. 55).
} 
capital como un todo. Ambos pasajes son tratados por Marx en los Grundrisse y en El capital con dos diferentes niveles de abstracción.

En los Grundrisse, Marx trata estos pasajes bajo la lógica de Hegel de lo uno y los muchos y, por lo tanto, en términos de repulsión y atracción. Con base en el primero, los muchos unos, esto es, los muchos capitales son puestos a través de la repulsión del uno, es decir, del capital en general, consigo mismo. En relación al segundo, el uno único, o sea, el capital como un todo, es puesto por medio de la atracción mutua de los muchos unos. Para el primer pasaje, Marx utiliza el doble sentido de repulsión que plantea Hegel: "la repulsión existente en $s i$ " y "la repulsión externa" ${ }^{46} \mathrm{y}$ refiere ambas a la competencia entre los muchos capitales:

También esta apariencia, que transgrede la proporción correcta, está fundada en la esencia del capital, según la cual, como lo analizaremos más pormenorizadamente en la competencia, los diversos capitales recíproca y totalmente indiferentes se repelen entre sí [...] (Marx, 1984a, p. 374, nota a pie de página).

Como el valor constituye la base del capital, y éste sólo existe, forzosamente, gracias al intercambio por un contravalor, el capital se repele necesariamente a sí mismo. Por ello es una quimera un capital universal, un capital que no tenga frente a sí capitales ajenos con los cuales intercambiar [...]. La repulsión recíproca de los capitales ya está implícita en él como valor de cambio realizado (Marx, 1984a, p. 375).

El primer sentido se refiere a la posición de los muchos capitales a través de la relación negativa del capital en general consigo mismo. Pero una vez que ya han sido puestos los muchos capitales, el segundo sentido se refiere a la repulsión recíproca externa de los muchos capitales entre sí. De acuerdo con Marx, es precisamente a través de la competencia así entendida que los muchos capitales

\footnotetext{
46 "Esta repulsión, en tanto es el poner a muchos unos, pero por medio de lo uno mismo, es el propio salir-fuera-de-sí de lo uno, pero hacia tales [seres] fuera de él, que son ellos mismos sólo unos. Es ésta la repulsión según el concepto, la repulsión existente en sí. La segunda repulsión es diferente de ésta, y es la que se asoma en seguida a la representación de la reflexión exterior, no como generación de los unos, sino sólo como el recíproco mantenerse alejados de unos que son presupuestos y ya presentes. Hay que ver, por lo tanto, cómo aquella repulsión existente en sí se determina [la] repulsión segunda, esto es, la exterior" (Hegel, 1968, p. 148).
} 
se ponen y se determinan recíprocamente entre sí como capitales socialmente existentes:

Por definición, la competencia no es otra cosa que la naturaleza interna del capital, su determinación esencial, que se presenta y realiza como acción recíproca de los diversos capitales entre sí; la tendencia interna como necesidad exterior. (El capital existe y sólo puede existir como muchos capitales; por consiguiente su autodeterminación se presenta como acción recíproca de los mismos entre sí) (Marx, 1984a, p. 366) (énfasis agregado).

Todos los momentos del capital que aparecen implícitos en él si se les considera según su concepto universal, adquieren una realidad autónoma, y se manifiestan, tan sólo cuando aquél se presenta realmente como muchos capitales (Marx, 1982b, p. 8)

La libre competencia es la relación del capital consigo mismo como otro capital, vale decir, el comportamiento real del capital en cuanto capital [...]. Lo inherente a la naturaleza del capital es puesto desde fuera [como una ${ }^{47}$ necesidad externa, por la competencia, que no es otra cosa sino que los muchos capitales se imponen, entre sí y a sí mismos, las determinaciones inmanentes del capital (Marx, 1982b, pp. 167-169).

En consecuencia, la noción de la competencia de Marx como acción recíproca de los diversos capitales entre sí puede concebirse como un proceso que opera, y cuyos efectos se dejan sentir, en dos niveles simultáneos de abstracción. El primero corresponde al nivel de la realidad esencial: éste se refiere a la reflexividad recíproca negativa de los muchos capitales entre sí a través de la cual ellos mismos, en la medida en que son concretamente diferentes uno de otro, se ponen y determinan como capitales esencialmente idénticos, es decir, como valores socialmente existentes que se autovalorizan. Aquí, ellos no difieren cualitativamente, sino sólo cuantitativamente. Ésta es su identidad dentro de su diferencia. El segundo corresponde al nivel de la apariencia concreta de la realidad: éste se refiere a la relación recíproca de los muchos capitales, mediante la

\footnotetext{
${ }^{47}$ En la traducción al español dice "con necesidad externa", mientras que en la traducción en inglés dice "as an external necessity", es decir, "como una necesidad externa". Se asume que lo que quiso decir Marx está mejor traducido por la versión en inglés; véase Marx (1973, pp. 650-651).
} 
cual, como capitales que son diferentes en muchos aspectos concretos, se oponen y rivalizan entre sí a fin de obtener su mayor valorización. Ésta, por el contrario, es su diferencia dentro de su identidad. ${ }^{48}$

En relación con el segundo pasaje, Marx considera, siguiendo a Hegel, que la repulsión recíproca de los muchos capitales es contrarrestada por su atracción. Su objetivo aquí es indicar que la multiplicidad de capital traspasa lo que Hegel (1968, p. 151) llama "un coincidir-consigo", el uno único, es decir, el capital como un todo social. Es a través de esta transposición que los muchos capitales son así unificados en una totalidad orgánica, "lo uno puesto como uno" (Hegel, 1968, p. 153), y cada uno de ellos se convierte en una parte alícuota del capital como un todo social -una totalidad social que, sin embargo, permanece arraigada en la repulsión de los muchos capitales.

Así, la competencia también se presenta como una relación contradictoria (en sentido dialéctico) entre el capital total y los muchos capitales que lo conforman, mediante la cual ambos se ponen y se determinan recíprocamente como capitales sociales existentes. El capital social total se pone como capital en su existencia en y para sí, y los muchos capitales como la existencia de las múltiples partes de él. Cada capital no es sólo uno de los muchos capitales, sino todos son uno y lo mismo. El capital social como un todo y los muchos capitales se constituyen así como momentos inseparables de una unidad orgánica. Sin la totalidad, las partes no existen, así como sin las partes, la totalidad no existe. El todo presupone las partes y las partes al todo. El todo y las partes, por lo tanto, son dos existencias de una misma unidad. ${ }^{49}$

En la sección segunda del tomo III de El capital, Marx concreta su noción de competencia en cuanto relación recíproca de los muchos capitales, en la

\footnotetext{
${ }^{48}$ Cabe señalar que en los textos de Marx y en la literatura al respecto el tratamiento de la competencia se enfoca básicamente en el nivel que hemos denominado de la apariencia concreta de la realidad. Por razones de espacio, no es posible tratar todas las implicaciones lógicas que supone la noción de competencia marxista en sus dos niveles de abstracción.

${ }^{49}$ En la Ciencia de la lógica, de Hegel, el todo y las partes forman un único y mismo concepto, se trata de una unidad dialéctica de opuestos, en la que el todo y las partes siempre se presuponen y se implican mutuamente. Es imposible definir el uno sin el otro; como hemos señalado en el caso de las mercancías, un elemento por sí sólo resulta incompleto, pues requiere la existencia de los otros elementos para autodefinirse; es, consecuentemente, contradictorio consigo mismo. Por tanto, no son los elementos los que resultan fundamentales, sino las relaciones que se definen entre ellos, en las cuales cada componente internaliza enteramente al otro. En la postura de totalidad orgánica, el todo forma sus partes y es simultáneamente formado por éstas. Como punto clave, el todo está presente en cada una de sus partes sin que éste pueda ser pensando como igual a la suma de sus partes; cada elemento es una expresión o representación del todo.
} 
posición del capital productivo en su totalidad, que es la única forma de capital que produce capital, donde cada uno de los muchos capitales es considerado como una rama particular del capital productivo, produciendo un mismo tipo de mercancía y cuya masa total de mercancías producidas es considerada "como una sola mercancía" que tiene "un solo precio" (Marx, 1976, p. 230).

Dado que la competencia se lleva a cabo a través de las relaciones de intercambio de las mercancías especificas que crean los diferentes capitales productivos particulares, es a través de sus relaciones mercantiles recíprocas que ellos se ponen y se determinan mutuamente como esencialmente idénticos y simultáneamente como concretamente diferentes. Esta doble posición remite, por un lado, al nivel de la realidad esencial, donde se presentan las determinaciones de las tasas uniforme y general de ganancia y los precios de producción, $\mathrm{y}$, por otro lado, al nivel de la apariencia concreta de la realidad, donde se presenta la determinación de las tasas diferenciales de ganancia y de los precios de mercado.

\section{Valor social actualizado y precio de producción}

En el plano o nivel de la realidad esencial, es a través de estas relaciones que los diferentes capitales productivos particulares se ponen socialmente como formas idénticas de valor como capital y, por lo tanto, a través de las cuales se reconocen mutuamente como iguales, difiriendo sólo cuantitativamente. En la medida en que expresan su posición cualitativa como capitales sociales existentes, sus relaciones son relaciones cualitativas que se manifiestan en una misma relación cuantitativa: la tasa uniforme de ganancia ${ }^{50}$ que es la medida de realización actualizada en el momento en que el capital aparece como una multiplicidad de capitales productivos particulares.

En cuanto la medida común de su realización, esta tasa expresa, siguiendo lo expuesto anteriormente, el cuanto de plusvalor en forma de ganancia que cada rama particular del capital productivo ha producido en proporción a su magnitud en un periodo determinado de tiempo e independientemente de la forma material de las mercancías que produce y de la composición técnica del capital utilizado para producirlas. Dado que la tasa uniforme de ganancia expre-

\footnotetext{
${ }^{50}$ La tasa uniforme de ganancia no puede ser lo mismo que la tasa promedio de ganancia: esta última sólo puede ser un promedio de las tasas diferenciales de mercado de ganancia de los capitales productivos.
} 
sa la posición de los diferentes capitales productivos particulares como socialmente idénticos, la forma dineraria del valor de las mercancías que cada uno de ellos produce se actualiza, adquiriendo una forma-precio correspondiente a esta tasa. Estos precios son lo que Marx denomina precios de producción. En este texto se sostiene, al contrario de las diversas interpretaciones al respecto,${ }^{51}$ que es a través de la determinación de los precios de producción que los valores de las mercancías elaboradas por todas las diferentes ramas particulares del capital productivo son ahora puestos, cualitativa y cuantitativamente, como valores sociales actualizados; y las cantidades de tiempo de trabajo objetivado en ellos son actualizadas como cuantos definitivos de tiempo de trabajo abstracto socialmente medido.

En los Grundrisse, Marx se refiere a la inversión dialéctica que resulta de considerar la competencia entre capitales individuales:

${ }^{51}$ La determinación de los precios de producción de Marx ha sido un tema muy controvertido, sobre el cual ha habido innumerables interpretaciones. Sin entrar a esta controversia, se reseñan sólo dos interpretaciones al respecto: por un lado, la marxista, que sostiene que los precios de producción no tienen existencia real y, por otro, la sraffiana, que sostiene que los precios de producción se pueden determinar sin necesidad del valor, del dinero y, por lo tanto, de los precios monetarios de las mercancías (véase nota 11). En relación a la primera interpretación, A. Shaikh (1992, p. 77) señala: "El proceso real de la competencia capitalista opera sobre la base de los precios de mercado reales, no sobre los precios proporcionales a los valores trabajo ni aun sobre los precios de producción [...]. No hay nunca un estado de equilibrio en que los precios de mercado 'converjan' en los precios de producción. De aquí que los precios de producción nunca existen como tales". Como, según Marx, los precios de producción dependen de la tasa uniforme o general de ganancia, esta interpretación implica que dichas tasas tampoco tienen existencia real y que, por lo tanto, las únicas existentes de verdad son las tasas diferenciales de mercado de ganancia. Con esto se confina a la realidad a una existencia puramente empírica, dejando de lado completamente la existencia esencial de la realidad capitalista. En cuanto que la interpretación sraffiana está fundamentada exclusivamente en las condiciones físicas de la producción, es decir, medios de producción materiales y cantidades de trabajos supuestamente homogéneos (pero que no explica cómo fueron homogeneizados) y salarios reales. Esta interpretación supone que la economía capitalista es fundamentalmente una economía físico-mercantil basada en el trueque, en la que el capital es considerado, en todo caso, una estructura física de producción, cuya medida es una tasa física de excedente. De aquí que el dinero esté ausente, o sea un simple numerario, en el cuerpo de la teoría y, por lo tanto, no se puedan conceptuar los precios, las ganancias y la(s) tasa(s) de ganancia en términos de la forma monetaria del capital. Con esto se limita a la realidad capitalista a la forma que corresponde al universo material general ahistórico, dejando de lado su forma de existencia específicamente social, es decir, la forma de valor, como dinero y capital. Sobre una presentación de las diferentes interpretaciones de la determinación de los precios de producción y las críticas a algunas de ellas, incluidas la neorricardiana tradicional y varias marxistas, véase Robles (2011, pp. 19-25 y 214-225). Para una crítica al problema de la medida del capital en Ricardo y en la interpretación sraffiana de Ricardo, véase las secciones 2.1 a 2.4 de Escorcia (2013, p. 60-71). 
En la competencia, la ley fundamental - que se desarrolla de manera diferente a la ley basada en el valor y el plusvalor-consiste en que el valor está determinado no por el trabajo contenido en él, o el tiempo de trabajo en que se le ha producido, sino por le tiempo de trabajo en que puede producírsele, o el tiempo de trabajo necesario para la reproducción. Sólo de esta manera el capital singular es puesto realiter [realmente] en las condiciones del capital en general, aunque la apariencia sea entonces como si hubiera quedado sin efecto la ley originaria. Pero sólo de esta manera el tiempo de trabajo necesario es puesto como determinado por el movimiento del capital mismo. Esta es la ley fundamental de la competencia. [...] En suma, aquí, todas las determinaciones se presentan a la inversa de lo que ocurría con el capital en general. Allí, el precio determinado por el trabajo; aquí, el trabajo determinado por el precio, etc., etc. El influjo de unos capitales individuales sobre los otros se origina precisamente en que tienen que comportarse como capital; la acción aparentemente autónoma de los individuos y sus colisiones no sujetas a reglas, son precisamente el poner de su ley general. El mercado adquiere aquí otro significado más. La acción recíproca de los capitales en cuanto entidades individuales se convierte precisamente en el ponerse de los mismos como generales (Marx, 1982b, pp. 175-176).

La competencia hace que la ley original basada en "el valor y el plusvalor", que se avanzó en el momento del capital en general, se invierta dialécticamente en la ley basada en los precios y ganancias. Como una inversión dialéctica, la ley original no es anulada, sino que es negada y superada y, por lo tanto, se mantiene como el fundamento de la ley de la competencia.

Retomando lo anterior en el contexto de la competencia de capitales en diferentes ramas del capital productivo, esta inversión tiene las siguientes implicaciones: a) el valor social de la mercancía elaborada por cada fracción particular del capital productivo total no se determina más "por el tiempo de trabajo en que se le produce", sino que se actualiza, determinándose por el tiempo de trabajo social abstracto que expresa su precio de producción; b) el precio de producción es, así, la actualización y, por tanto, la negación y superación del precio directo del valor social de las mercancías del mismo tipo creado en determinada rama, ${ }^{52}$ cuya determinación se presentó en forma aislada de la totalidad de las ramas del capital productivo en el momento del capital en general, en el primer

${ }^{52}$ El valor social de las mercancías, tal como se define en el tomo i de El capital, se concreta como valor de mercado en el tomo III. 
tomo de El capital, y c) es sólo por medio de la competencia, entendida como acción recíproca de los muchos capitales en el mercado, que todas las diferentes fracciones del capital productivo son puestas en las condiciones de capital en general, es decir, "como entidades generales" realmente existentes.

De ello, su puede afirmar adicionalmente que, por un lado, debido a la unidad dialéctica de forma y contenido que subyace en los diferentes momentos en que el concepto de capital se presenta en El capital, los procesos de determinación de los precios de producción de las mercancías y de la reducción cuantitativa y cualitativa del trabajo objetivado en ellas como trabajo social abstracto sólo pueden entenderse como dos procesos que se realizan simultáneamente entre sí. Por otro lado, contrariamente a lo que Marx mismo y muchos economistas políticos marxistas sostienen, la actualización de la determinación de los valores sociales de todo tipo de mercancías como productos de los muchos y diversos capitales productivos no puede entenderse como resultado de los promedios ponderados de la suma de los tiempos de trabajo individuales gastados dentro de su rama de producción, sino como el tiempo de trabajo necesario que es (im)puesto o validado socialmente por el capital productivo como un todo $^{53} \mathrm{y}$, en consecuencia, la determinación de los precios de producción no implica transferencias de valor o plusvalía entre las diferentes ramas del capital productivo. ${ }^{54}$

En el cuadro 3 se representa el proceso de determinación del valor social actualizado de mercancías heterogéneas por mediación de sus precios de producción, suponiendo que hay sólo dos ramas de capital productivo que elaboran, cada una, un tipo específico de mercancías, $C_{1}$ o $C_{2}$, con diferentes métodos de producción, lo que se expresa en que utilizan: a) diferentes cantidades de mercancías como medios de producción $\left(C_{11}\right.$ y $C_{21}$ en la elaboración de $C_{1}, \mathrm{y} C_{12}$ y $C_{22}$ en la de $\left.C_{2}\right)$, las cuales entran a la producción con sus valores sociales $\left(\lambda^{\circ} C_{i}\right)$ tal como fueron determinados al interior de cada rama y $b$ ) diferentes cantidades de

53 "Si el valor de las mercancía está determinado por el tiempo de trabajo necesario contenido en ellas, y no por el tiempo de trabajo contenido en ellas en forma general, es el capital el primero que realiza esta determinación" (Marx, 1976, p. 105).

${ }^{54}$ La conceptualización de Marx de las transferencias de valor y/o plusvalor entre las diferentes ramas del capital productivo debidas a la determinación de los precios de producción se encuentra, entre otros, en el siguiente pasaje: "Por lo tanto, en el caso de las mercancías producidas por el capital II, su valor sería inferior a su precio de producción, y en el de las mercancías producidas por el capital [III] el precio sería menor que el valor; sólo en el caso de los capitales I de los ramos de la producción cuyas composición es, casualmente, la misma del promedio social, el valor y el precio de producción serían iguales" (Marx, 1976, p. 207). 
tiempo de trabajo directo socialmente requerido para su producción en sus ramas respectivas $\left(1^{\circ}{ }_{1}\right.$ y $\left.1_{2}^{\circ}\right)$.

Cuadro 3. Determinación de los valores sociales actualizados de las mercancías por mediación de sus precios de producción

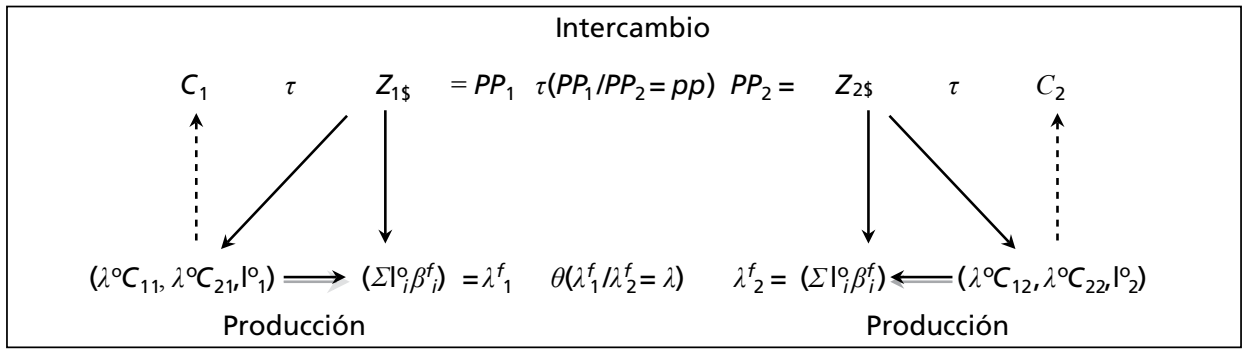

Donde: $\mathrm{I}_{i}{ }_{i} \quad=$ el tiempo de trabajo social objetivado directamente en la rama que produce las mercancías $C_{i}$;

$\lambda^{\circ} C_{i}=$ el valor social de la mercancía $\mathrm{i}$, tal como es determinado en la rama que la produce, el cual es equivalente a su precio directo, $P_{D i}$;

$C_{i j}=$ la cantidad de la mercancía i como medio de producción requerido para elaborar la mercancía j;

$\tau=$ la relación de igualación de las mercancías en el espacio monetario;

$Z_{i \$} \quad=$ las unidades monetarias que representan el precio de producción, $P P_{i}$, de $C_{i}$;

$P P_{i}=$ el precio de producción de la mercancías $C_{\mathrm{j}}$;

$\longrightarrow=$ las relaciones de determinación del valor social actualizado de las mercancías por mediación de sus precios de producción;

$p p=$ el precio de producción relativo;

$\mathrm{I}_{i}{ }_{i}=$ el tiempo de trabajo directo socialmente requerido para producir la mercancía $i$;

$\Sigma \mathrm{I}^{\circ}{ }_{i}=$ los tiempos de trabajo social directos e indirectos objetivados en la rama que produce las mercancías $C_{i}$;

$\beta^{f}{ }_{i}=$ los coeficientes de reducción finales mediante los cuales las unidades de tiempo de trabajo social se transforman en unidades de tiempo de trabajo social actualizado;

$\lambda^{f}{ }_{i}=$ el valor social actualizado de las mercancías $C_{i}$, que es igual a determinadas cantidades de tiempo de trabajo social actualizado, directa e indirectamente, objetivadas en ellas $\left(\Sigma \mathrm{I}^{\mathrm{O}}{ }_{i j} \beta^{f}{ }_{i}\right)$;

$\theta=$ la relación de igualación de las mercancías en el espacio del valor social actualizado;

$\lambda=$ el valor social actualizado relativo de las mercancías.

En cuanto que los capitales productivos que componen las diversas ramas de la producción sólo se ponen como socialmente existentes por mediación de su acción recíproca, es decir, de la competencia en la esfera del intercambio y, por lo tanto, por mediación de las relaciones recíprocas de las mercancías que producen, lo que se expresa en la tasa uniforme de ganancia $(r)$, las formas di- 
nerarias de valor que las mercancías $\left(C_{i}\right)$ adquieren correspondientes a esta tasa son las de los precios de producción $\left(P P_{i}\right)$. En este sentido, como se muestra en el cuadro 3, es por medio de la determinación de los precios de producción $\left(P P_{i}\right)$ de las mercancías $\left(C_{i}\right)$ que sus valores sociales $\left(\lambda^{\circ} C_{i}\right)$, tal como son determinados en la rama que las produce, se ponen como valores sociales actualizados o definitivos $\left(\lambda^{f} C_{i}\right)$, lo que implica, a su vez, que las cantidades de tiempo de trabajo social objetivadas directa e indirectamente en su producción $\left(\Sigma 1^{\circ}{ }_{i}\right)$ se ponen, cualitativa y cuantitativamente, como cuantos de tiempo de trabajo social actualizado o definitivo $\left(\Sigma 1_{i}^{0} \beta_{i}^{f}\right)$. Los coeficientes de reducción finales $\left(\beta_{i}^{f}\right)$ expresan esta transformación. Esta posición representa, así, la superación (o la transformación) del espacio del valor social -es decir, del tiempo de trabajo social objetivado en las mercancías tal como es determinado al interior de cada una de las ramas particulares del capital productivo- por el (en el) espacio del valor social actualizado - es decir, el tiempo de trabajo social actualizado que se objetiva en ellas tal como es finalmente determinado al considerar el capital productivo social en su totalidad.

El precio de producción, $P P_{i}$, de las mercancías, $C_{i}$, producidas por cualquier rama particular de capital productivo, $i$, puede representarse mediante la siguiente fórmula:

$$
P P_{i}=\left(c_{i}+v_{i}\right)(1+r)=c_{i}+v_{i}+g_{i} \Rightarrow\left(\Sigma 1^{\mathrm{o}}{ }_{M P-i} \beta^{f}{ }_{i}\right)+\left(\mathrm{l}_{i}^{\mathrm{o}} \beta^{f}{ }_{i}\right)=\lambda{ }_{M P-i}^{f}+\left(1^{\mathrm{o}}{ }_{i} \beta^{f}\right)=\lambda^{f}{ }_{i}
$$

donde $c_{i}=$ el capital constante, equivalente a los precios de producción de los medios de producción utilizados; $v_{i}=$ capital variable; $r=$ la tasa uniforme de ganancia; $g_{i}=$ ganancias $=\left(c_{i}+v_{i}\right) r$, que son equivalentes al plusvalor producido, $p v_{i} ; 1_{M P-i}^{\circ}=$ el tiempo de trabajo social objetivado en los medios de producción; $\beta_{i}^{f}=$ los coeficientes de reducción finales mediante los cuales las unidades de tiempo de trabajo social, $1_{i}^{\circ}$, se transforman en unidades de tiempo de trabajo social actualizado; $\Sigma \mathrm{l}^{\circ}{ }_{M P_{-}-} \beta_{i}^{f}=\lambda{ }_{M{ }^{f}-i}$, es decir, los tiempos de trabajo social actualizados que se objetivan en los medios de producción, equivalentes a sus valores sociales actualizados $\mathrm{y}$, por lo tanto, proporcionales a $c_{i} ; 1_{i}^{\circ} \beta^{f}{ }_{i}=$ el tiempo de trabajo social actualizado directamente objetivado en las mercancías, proporcionalmente igual a $\left(v_{i}+p v_{i}\right)=\left(v_{i}+g_{i}\right)$.

Por otro lado, la relación cuantitativa que manifiesta la posición cualitativa del capital productivo como un todo se refiere a su medida específica de realización: la tasa general de ganancia del capital productivo como un todo, que, en la medida en que es el resultado "del grado de explotación del trabajo 
global por el capital global" (Marx, 1976, p. 248), expresa el cuanto de valor excedente o plusvalor total en forma de ganancia que éste ha producido en un periodo de tiempo determinado. Así, "la tasa general de ganancia es el momento donde el capital social global establece su unidad consigo mismo" (Arthur, 2001, p. 144) y donde, como tal, se enfrenta a su propio otro como un todo, es decir, el trabajo productivo como un todo. Es en este contexto que Marx señala que para el capital productivo social total "el precio de producción es igual al valor" (Marx, 1976, p. 208).

\section{Valor social actualizado y precio de mercado}

En el plano o nivel de la apariencia concreta de la realidad, la competencia entre los capitales productivos hace que las formas dinerarias de las mercancías elaboradas en las diferentes ramas y la tasa de ganancia correspondiente a ellas se desvíen de sus precios de producción y de la tasa uniforme de ganancia, respectivamente. Estas formas dinerarias son los precios de mercado de las diferentes mercancías y la tasa que corresponde a la rama particular del capital productivo que las crea es su tasa de ganancia de mercado, es decir, su medida concreta de realización.

Como desviaciones de sus precios de producción, los precios de mercado de las mercancías representan ciertas cantidades de valor social actualizado, es decir, ciertas cantidades de tiempo de trabajo social actualizado, que pueden ser mayores, menores o iguales que aquellas que expresan sus precios de producción. Así, las ventas de las mercancías a sus precios de mercado, a diferencia de lo que ocurre con los precios de producción, implican transferencias de plusvalor en forma de ganancias, desde las ramas cuyos precios de mercado están por debajo de sus precios de producción hacia las ramas en las que los primeros están por encima de los segundos. En la medida en que existen, los precios de producción y la tasa uniforme de ganancia constituyen los centros de gravedad alrededor de los cuales fluctúan los precios de mercado y las tasas de ganancia de mercado, respectivamente. ${ }^{55}$

Cabe señalar que debido a que los precios de producción y las tasas uniformes de ganancia no pueden detectarse directamente, la única manera de cap-

${ }^{55}$ Es este sentido que Marx (1976, p. 227) sostiene que los precios de producción son "el centro en torno al cual giran los precios del mercado diarios, y hacia el cual se nivelan en determinados periodos". 
tarlos es a través de las medias ponderadas de los precios de mercado y de las tasas de ganancia de mercado, respectivamente. Estas cifras promedio pueden denominarse precio de producción de mercado o promedio y tasa media de ganancia, respectivamente, y las ganancias correspondientes a ellos, ganancia media. Aunque no coincidan con los precios de producción y la tasa uniforme de ganancia, estas cifras promedio no son, dice Marx, "en modo alguno, de importancia meramente teórica, sino de importancia práctica para el capital, cuya inversión se calcula según las oscilaciones y compensaciones en un lapso más o menos determinado" (Marx, 1976, p. 240). Lo que queda claro aquí, es que estas cifras promedio no necesariamente corresponden a los precios de producción o a la tasa uniforme de ganancia.

Dado que los capitales individuales dentro de cualquier rama del capital productivo operan con composiciones orgánicas de capital que difieren entre sí, y que cada rama elabora el mismo tipo de mercancías que tienen el mismo precio de mercado, la tasa de ganancia de cada uno de ellos es su tasa individual de ganancia de mercado, es decir, su medida individual de realización, que difiere no sólo de las de los otros capitales individuales, sino también de la tasa de ganancia de mercado de la rama productiva particular. Esto se muestra por el hecho de que, mediante la venta de sus mercancías al precio del mercado, cualquier capital individual obtiene una cierta ganancia, a través de la cual se calcula su propia tasa individual de ganancia de mercado, cuya magnitud puede ser mayor, menor o igual que la tasa de mercado de la rama particular del capital productivo de la que éste es una fracción.

Como cualquier capital individual que es esencialmente un capital en general, cuyo objetivo fundamental es valorizarse a sí mismo y, por lo tanto, acumular tanto como sea posible, éste no intenta extraer para su propio "capital adelantado con vistas a la producción" simplemente "el mismo plusvalor o ganancia que cualquier otro capital de la misma magnitud o pro rata a su magnitud, cualquiera que sea el ramo de la producción en el cual se le haya empleado" (Marx, 1976, p. 240), sino que, por el contrario, rivaliza con los otros capitales individuales a fin de obtener una mayor valorización. Es en este nivel más concreto de la competencia donde se revela que cada capital individual pretende suplantar a sus competidores y excluirlos del mercado. ${ }^{56}$ Para ello, cada uno de los capitales

56 " [...] acorde con la naturaleza de la producción capitalista [...] cada capital [individual] de por sí trata de capturar en el mercado el mayor sitio posible y de desplazar, de eliminar a sus copartícipes: competencia entre capitales" (Marx 1980b, pp. 446-447). 
individuales intenta introducirse a sí mismo y utilizar algunos de los instrumentos más poderosos para ello, como la innovación tecnológica y el crédito.

\section{CONCLusiones}

Se puede decir, por todo lo expuesto anteriormente, que la circulación mercantil simple (M-D.D-M) -que pertenecía al primer momento de la presentación- no puede conceptuarse más como la apariencia inmediata de la circulación capitalista, carente de fundamento, sino como la apariencia fundamentada de la esfera de la circulación ( $M{ }^{\prime}-D$ '.D-M) del circuito de la reproducción del capital (D-M... P....M'-D'.D-M...P....'-D'). De esta manera, cualquier mercancía particular que ahora aparezca en la superficie de los fenómenos "se distingue de la mercancía de la que la producción capitalista parte como elemento". En verdad, como producto de un determinado capital productivo individual particular, ésta se manifiesta no como simple mercancía, sino más bien "como parte no sólo real, sino también [como parte] de la producción total" y aparece "como exponente de una determinada parte del capital y de la plusvalía creada por él” (Marx, 1980c, p. 98). Así, la mercancía, el punto de partida de la presentación en El capital, y sus determinaciones de forma de valor son fundamentadas sólo regresivamente hasta el momento en que el capital se pone como muchos capitales, esto es, hasta el momento de la competencia; y la mercancía puede ser así concebida como la unidad de sus diversas determinaciones como producto de capital.

A lo largo de los cuatro momentos, esto es, desde la forma de existencia inmediata hasta la multiplicidad del capital con la que se llega a los precios de producción y los precios de mercado, hay dos elementos que deben ser subrayados como una síntesis de la posición expuesta: en primer lugar, a diferencia de la interpretación dominante, propia tanto de la escuela tradicional marxista como de la sraffiana, se considera que el valor no es una sustancia establecida plenamente en la producción, sino que su posición social sucede en el momento del intercambio, es decir, en la circulación. Esto significa que sólo a partir de la relación dialéctica entre producción y circulación puede determinarse el valor, planteándose consecuentemente que no existe una relación unidireccional que va de valores a precios de producción, sino una determinación reciproca entre valor y precio. En segundo lugar, en relación a los problemas de la transformación de valores a precios y de la reducción de los trabajos privados heterogéneos a trabajo social abstracto, se ha considerado que ambos se implican mutuamente y deben ser resueltos de manera simultánea, debido a que en la relación valor-precio, 
la forma-precio es la forma general de existencia dineraria del valor social de las mercancías y los valores sociales representan siempre una determinada cantidad de tiempo de trabajo social abstracto. Tras el análisis del desenvolvimiento dialéctico de la forma-precio y de la relación valor-precio en los diferentes momentos de la estructura lógica del concepto de capital, la propuesta de solución puede ser resumida así: si la forma-precio es la forma general de existencia del valor social, los valores sociales actualizados o definitivos de las mercancías como productos de capitales son aquéllos puestos por mediación de la determinación de sus precios de producción y, por tanto, los diferentes tiempos de trabajos privados objetivados en ellas son reducidos a tiempos de trabajo social abstracto definitivo por medio de dicha determinación. Esto representa una inversión en términos dialécticos de la relación de determinación entre valor y precio: en el cuarto momento de la presentación, los precios no aparecen determinados por el trabajo, sino, por el contrario, los tiempos de trabajo social abstracto definitivos y, por tanto, los valores sociales actualizados aparecen determinados por los precios.

\section{REFERENCIAS BIBLIOGRÁFICAS}

Arthur, Christopher, J. (1993), "Hegel's Logic and Marx's Capital", en Fred Moseley (ed.), Marx's Method in Capital. A Reexamination, USA, Humanities Press, pp. 63-87.

- (2001), "Capital, Competition and Many Capitals", en Martha Campbell y Geert Reuten (eds.), The Culmination of 'Capital': Essays on Volume Three of Marx's 'Capital', UsA, Palgrave.

Böhm-Bawerk, Eugen (1949), Karl Marx and the close of his system, New York, Augustus M. Kelley.

Dostaler, Gilles (1980), Valor y precio. Historia de un debate, México, Editorial Terra Nova.

Duménil, Gérard, y Lévy, Dominique (1993), The Economics of the Profit Rate, Aldershot/Brookfield, Edward Elgar.

Dussel, Enrique (2005), "Hegel, Schelling y el plusvalor", en Mario L. Robles Báez (comp.), Dialéctica y capital. Elementos para una reconstrucción de la crítica de la economía política, México, UAM-Xochimilco.

Escorcia, Roberto (2013), Fundamentación lógica del dinero y del proceso de financiamiento desde el concepto de capital, tesis de Doctorado en Ciencias Económicas, Universidad Autónoma Metropolitana. 
Gaete, Arturo (1995), La lógica de Hegel. Iniciación a su lectura, Buenos Aires, Argentina, Edicial.

Gortari, Eli de (1970), El método dialéctico, México, Editorial Grijalbo.

Hegel, Georg Wilhelm Friedrich (1968), Ciencia de la lógica, 5ª ed., Buenos Aires, Solar/Hachete.

- (1974), Enciclopedia de las Ciencias Filosóficas, México, Juan Pablos Editor.

(1997), Enciclopedia de las Ciencias Filosóficas, $7^{\mathrm{a}}$. ed., Sepan Cuantos 187, México, Porrúa.

Inwood, Michael (1993), A Hegel Dictionary, Oxford/Cambridge, Blackwell Publishers. Marx, Karl (1970), A Contribution to the Critique of Political Economy, Moscow, Progress Publishers.

- (1973), Grundrisse. Foundations of the Critique of Political Economy (Rough Draft), London, Penguin Books.

- (1976), El capital. Crítica de la economía política, t. III, vol. 6, México, Siglo XXI Editores.

- (1978), El capital. Crítica de la economía política, t. II, vol. 4, Siglo XXI Editores.

— (1980a), Contribución a la crítica de la economía política, México, Siglo XXI Editores.

— (1980b), Teorías sobre la plusvalía II, México, Fondo de Cultura Económica.

— (1980c), Teorías sobre la plusvalía III, México, Fondo de Cultura Económica.

- (1982a), El capital. Crítica de la economía política, t. I, vol. 1, México, Siglo XXI Editores.

- (1982b), Elementos fundamentales para la crítica de la economía política, vol. 2, 9a . ed., México, Siglo xxi Editores.

- (1984a), Elementos fundamentales para la crítica de la economía política, vol. 2, $13^{\mathrm{a}}$. ed., México, Siglo xxi Editores.

- (1984b), El capital. Crítica de la economía política, t. I, vol. 2, México, Siglo XXI Editores.

(1984c), El capital. Crítica de la economía política, t. I, vol. 3. México, Siglo XXI Editores.

Marx, Karl, y Engels, Friedrich (1983), Cartas sobre El capital, La Habana, Editora Política.

Meaney, Mark E., (2002) Capital as Organic Unity. The Role of Hegel's 'Science of Logic' in Marx's 'Grundrisse'. Dordrecht/Boston/London, Kluwer Academic Publisher.

Murray, Patrick (2005), “La teoría del valor trabajo 'verdaderamente social' de Marx: el 
trabajo abstracto en la teoría marxista del valor", en Mario L. Robles Báez (comp.), Dialéctica y capital. Elementos para una reconstrucción de la crítica de la economía política, México, UAM-Xochimilco.

Pietranera, Giulio (1977), "La estructura lógica de El capital", en Maurice Dobb et al., Estudios sobre el capital, México, Siglo xxi Editores.

Robinson, Joan (1968), Introducción a la economía marxista, México, Siglo XXI Editores.

- (1976), Relevancia de la teoría económica, España, Ediciones Martínez Roca.

Robles, Mario L. (2001), “Acerca del trabajo abstracto como una abstracción socialmente determinada", Economía: Teoría y Práctica, 0(14), pp. 157-190.

(2005) (comp.), Dialéctica y capital. Elementos para una reconstrucción de la crítica de la economía política, México, UAM-Xochimilco.

_ (2008), "La tasa de ganancia en la conceptualización del capital de Marx: Una reinterpretación”, en Sergio Cámara, Etelberto Ortiz y Mario Robles (comps.), Reproducción y capital. Equilibrio y desequilibrio desde una perspectiva crítica de la economía, México, Ediciones Eón/UAM-A.

- (2011), Marx: lógica y capital. La dialéctica de la tasa de ganancia y la formaprecio, México, UAM-Xochimilco.

Samuelson, Paul (1971), "Undestanding the Marxian Notion of Exploitation: A Summary of the So-Called Transformation Problem between Marxian Values and Competitive Prices", Journal of Economic Literature, 9(2), pp. 399-431.

Shaikh, Anwar (1992), "Value and Value Transfer: a Comment on Itoh", en Bruce Roberts y Susan Feiner (eds.), Radical Economics, Boston, USA, Kluwer Academic Publishers.

Smith, Adam (1958), Investigación sobre la naturaleza y causas de la riqueza de las naciones, $2^{\mathrm{a}}$. ed., México, Fondo de Cultura Económica.

Steedman, Ian (1985), Marx, Sraffa y el problema de la transformación, México, Fondo de Cultura Económica. 